\title{
Initial Hepatitis C Virus Infection of Adult Hepatocytes Triggers a Temporally Structured Transcriptional Program Containing Diverse Pro- and Antiviral Elements
}

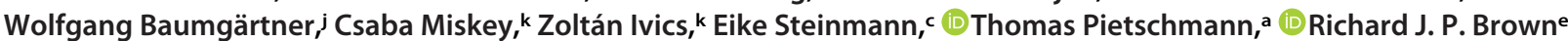 \\ alnstitute of Experimental Virology, TWINCORE, Centre for Experimental and Clinical Infection Research, Hannover, Germany \\ bHeinrich Pette Institute, Leibniz Institute for Experimental Virology, Hamburg, Germany \\ cDepartment of Molecular and Medical Virology, Bochum, Germany \\ dEuropean Virus Bioinformatics Center (EVBC), Jena, Germany \\ eDivision of Veterinary Medicine, Paul Ehrlich Institute, Langen, Germany \\ fDepartment of General, Visceral and Transplant Surgery, Hannover Medical School, Hannover, Germany \\ gGerman Centre for Infection Research (DZIF), Partner Site Hannover-Braunschweig, Hannover, Germany \\ nDepartment of Gastroenterology and Hepatology, University Hospital Essen, University Duisburg-Essen, Essen, Germany \\ 'Helmholtz Institute for RNA-Based Infection Research (HIRI), Helmholtz Centre for Infection Research (HZI), Würzburg, Germany \\ iDepartment of Pathology, University of Veterinary Medicine Hannover, Hannover, Germany \\ kDivision of Medical Biotechnology, Paul Ehrlich Institute, Langen, Germany
}

(D) Birthe Tegtmeyer, ${ }^{a}$ (D) Gabrielle Vieyres, ${ }^{b}$ Daniel Todt,,${ }^{c, d}$ Chris Lauber, ${ }^{a}$ Corinne Ginkel, ${ }^{a}$ Michael Engelmann, ${ }^{c}$ Maike Herrmann, ${ }^{e}$

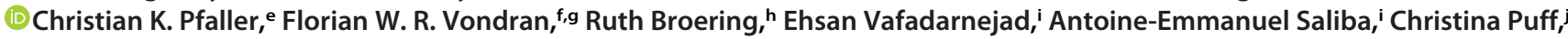

ABSTRACT Transcriptional profiling provides global snapshots of virus-mediated cellular reprogramming, which can simultaneously encompass pro- and antiviral components. To determine early transcriptional signatures associated with hepatitis $\mathrm{C}$ virus $(\mathrm{HCV})$ infection of authentic target cells, we performed ex vivo infections of adult primary human hepatocytes (PHHs) from seven donors. Longitudinal sampling identified minimal gene dysregulation at $6 \mathrm{~h}$ postinfection (hpi). In contrast, at $72 \mathrm{hpi}$, massive increases in the breadth and magnitude of HCV-induced gene dysregulation were apparent, affecting gene classes associated with diverse biological processes. Comparison with HCV-induced transcriptional dysregulation in Huh-7.5 cells identified limited overlap between the two systems. Of note, in $\mathrm{PHH}, \mathrm{HCV}$ infection initiated broad upregulation of canonical interferon (IFN)-mediated defense programs, limiting viral RNA replication and abrogating virion release. We further find that the constitutive expression of IRF1 in PHHs maintains a steady-state antiviral program in the absence of infection, which can additionally reduce HCV RNA translation and replication. We also detected infection-induced downregulation of $\sim 90$ genes encoding components of the elF2 translation initiation complex and ribosomal subunits in $\mathrm{PHHs}$, consistent with a signature of translational shutoff. As HCV polyprotein translation occurs independently of the elF2 complex, this process is likely proviral: only translation initiation of host transcripts is arrested. The combination of antiviral intrinsic and inducible immunity, balanced against proviral programs, including translational arrest, maintains HCV replication at a low level in PHHs. This may ultimately keep HCV under the radar of extrahepatocyte immune surveillance while initial infection is established, promoting tolerance, preventing clearance, and facilitating progression to chronicity.

IMPORTANCE Acute HCV infections are often asymptomatic and therefore frequently undiagnosed. We endeavored to recreate this understudied phase of HCV infection using explanted PHHs and monitored host responses to initial infection. We detected temporally distinct virus-induced perturbations in the transcriptional landscape, which were initially narrow but massively amplified in breadth and magnitude over time. At $72 \mathrm{hpi}$, we detected dysregulation of diverse gene programs, concurrently
Citation Tegtmeyer B, Vieyres G, Todt D, Lauber C, Ginkel C, Engelmann M, Herrmann M Pfaller CK, Vondran FWR, Broering R, Vafadarnejad E, Saliba A-E, Puff C, Baumgärtner W, Miskey C, Ivics Z, Steinmann E, Pietschmann T, Brown RJP. 2021. Initial hepatitis C virus infection of adult hepatocytes triggers a temporally structured transcriptional program containing diverse pro- and antiviral elements. J Virol 95:e00245-21. https://doi.org/10.1128/ JVI.00245-21.

Editor J.-H. James Ou, University of Southern California

Copyright $\odot 2021$ American Society for Microbiology. All Rights Reserved.

Address correspondence to Richard J. P. Brown, Richard.Brown@pei.de.

Received 12 February 2021

Accepted 25 February 2021

Accepted manuscript posted online

3 March 2021

Published 26 April 2021 
promoting both virus clearance and virus persistence. On the one hand, baseline expression of IRF1 combined with infection-induced upregulation of IFN-mediated effector genes suppresses virus propagation. On the other, we detect transcriptional signatures of host translational inhibition, which likely reduces the processing of IFNregulated gene transcripts and facilitates virus survival. Together, our data provide important insights into constitutive and virus-induced transcriptional programs in $\mathrm{PHH}$ and identify simultaneous antagonistic dysregulation of pro- and antiviral programs, which may facilitate host tolerance and promote viral persistence.

KEYWORDS hepatitis C virus (HCV), RNA-seq, primary human hepatocytes, IFN regulatory factor 1 (IRF1), IFN signaling, elF2 signaling, translational shutoff

D espite the development of effective antiviral therapies, hepatitis $C$ virus (HCV) remains a global health burden and still chronically infects around 71 million people worldwide. HCV is a positive-stranded RNA virus of the Flaviviridae family with a tropism restricted to human hepatocytes (1). The development of cell culture systems has yielded insights into HCV-host interactions. For instance, lipid metabolic reprogramming favorable to the constitution of the viral replication organelle (2) and stress responses (3) were reported upon HCV infection. These dysregulations facilitate viral propagation but also the development of pathogenesis in chronic infection, including liver steatosis (4). Balanced against this, HCV infection elicits a classical innate immune response that obeys rules that are conserved across viruses (5). Virus infection is sensed via diverse pattern recognition receptors (PRRs), which trigger signaling cascades involving the nuclear translocation of interferon (IFN) regulatory factors (IRFs) and NF-KB (nuclear factor $\mathrm{kB}$ ). These transcription factors (TFs) activate the expression of target genes, including IFNs, which in turn activate the transcription of a panel of IFN-regulated genes (IRGs) in a paracrine and autocrine manner (6). Many of these IRGs have known antiviral effects (7). Importantly, these host protective responses are dampened by the virus, for example, by the viral protease NS3-4A, which cleaves several key molecules in the IFN induction pathway, including the adaptor MAVS (5).

Many of these changes, whether metabolic reprogramming to facilitate viral replication or defense reactions, are accompanied or caused by transcriptional alterations of the infected cell $(8,9)$. Transcriptome-wide studies of HCV infection mostly focused so far on cell lines, in particular on the Huh-7.5 hepatoma cell line (10), which represents a robust in vitro infection model (e.g., see references 8 and 9). Transcriptional profiling of liver biopsy specimens from chronically infected patients was also performed (11). Previous studies in primary human hepatocytes ( $\mathrm{PHHs}$ ) were based on reverse transcription-quantitative PCR (RT-qPCR) or microarrays and restricted to specific gene subsets, in particular focusing on innate immunity (12-14). Responses to HCV infection in infected human hepatocytes at nearly single-cell resolution were described. However, these cells were derived from fetal liver cells rather than being directly explanted from an adult liver (15) and have an immature phenotype (16). Additionally, the initial phase of infection directly preceding infection was not captured.

In this report, we used RNA sequencing (RNA-seq) (17) to analyze the host transcriptional landscape directly after HCV infection of adult PHHs. Infection of human adult hepatocytes plated from liver resections of HCV-negative patients opens the unique possibility to study early time points directly after infection of natural HCV target cells, without contamination from other liver cell types (18), which could blur transcriptional signals. We compared the responses at $6 \mathrm{~h}$ postinfection (hpi) and $72 \mathrm{hpi}$ in order to reflect the spatial transitioning of viral replication complexes from ribosomes (19) to the endoplasmic reticulum (ER) (20). In parallel, we also performed transcriptional profiling of highly permissive Huh-7.5 cells, which are widely used to propagate HCV in vitro, and directly compared HCV-induced gene dysregulation in the two systems for the first time. 
A

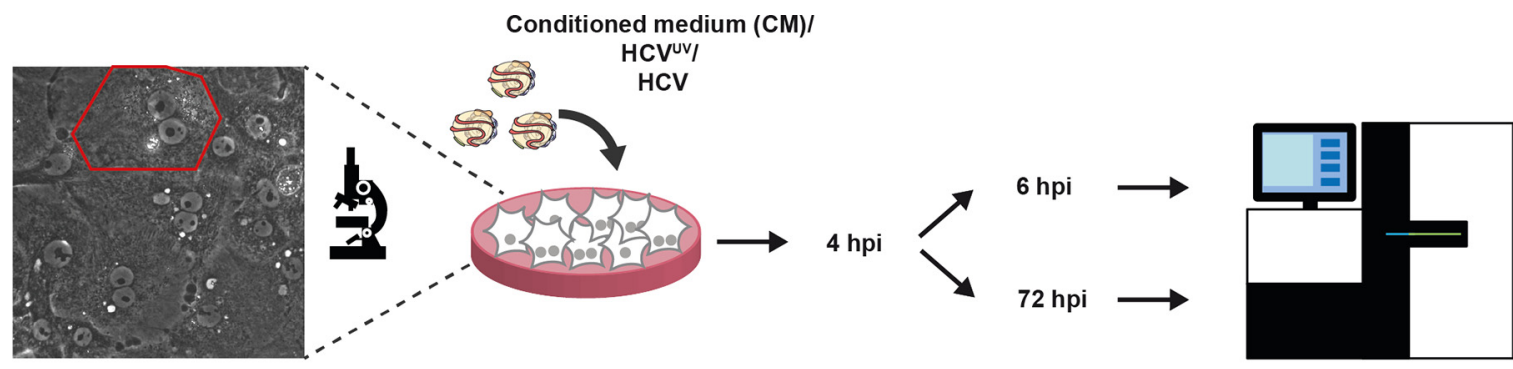

PHH inoculation Medium change Total RNA isolation

B

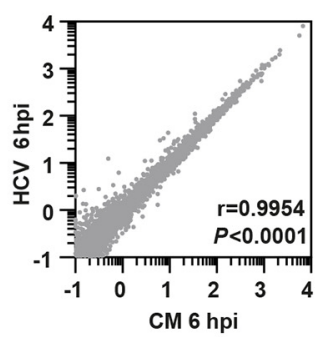

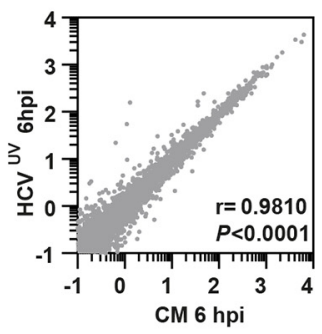

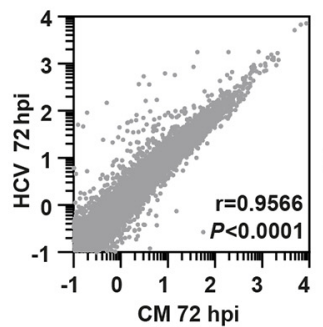

CM 72 hpi

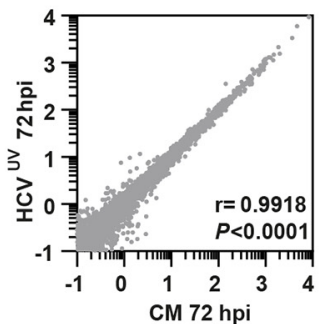

RNA-Seq

C

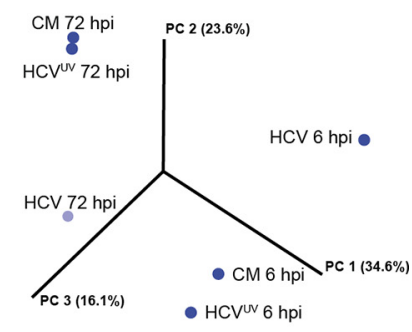

D

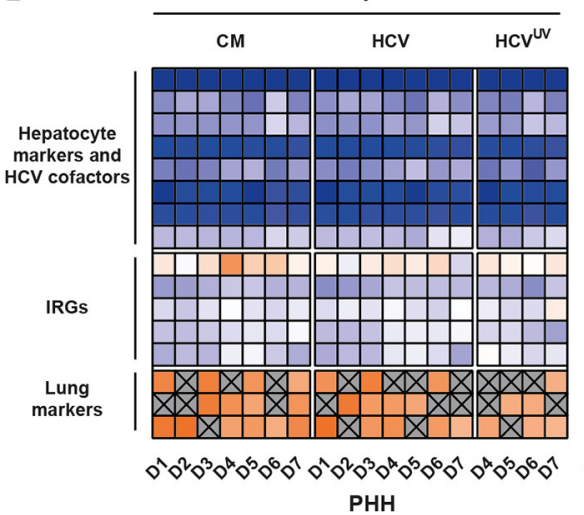

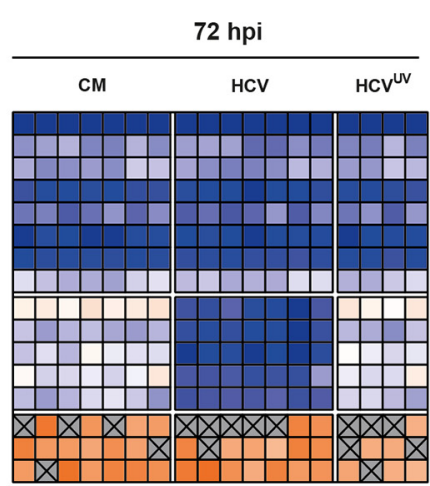

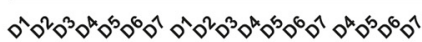

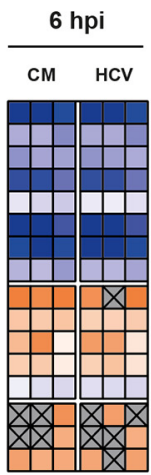

Huh-7.5

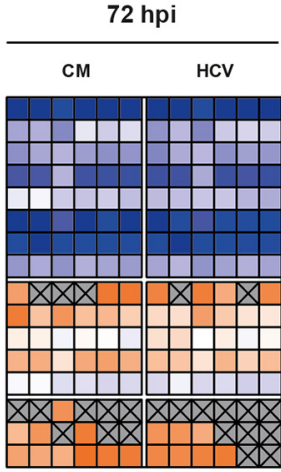

Huh-7.5

\section{ALB $\square>2.5$} CD81 1 SCARB1 $\begin{array}{lll}\text { SCARB1 } & 2 \\ \text { CLDN1 } & 1 & 1 \\ \text { OLO } & \log _{10}\end{array}$ OCLN - $0 \log _{10}$ $A P O E$
$P P I A$ PI4KA $\square$ DO DSADRA IFIT1 OAS3 PLSCR1 SFTPA1 SFTPA2 SFTPB

$\mathrm{PHH}$

FIG 1 Experimental protocol and RNA sequencing (RNA-seq) data validation. (A) Schematic of the experimental protocol. (B) Visualization of global transcriptional changes induced by HCV infection ( $n=7$ donors) or treatment with UV-inactivated HCV (HCV ${ }^{U V}$ ) ( $n=4$ donors). For individual plots, average RPKM $\left(\log _{10}\right)$ values for all detected transcripts from CM-treated cells are plotted on the $x$ axes, with corresponding values from HCV-infected and HCVUV treated cells plotted on the $y$ axes, respectively. Pearson's $r$ and $P$ values for each comparison are inset. (C) Three-dimensional principal-component analysis (PCA) from primary human hepatocyte (PHH) donor 4. Samples are distinguishable depending on their time point and infection status. (D) Control gene expression levels in PHHs and Huh-7.5 cells at 6 and $72 \mathrm{~h}$ postinfection (hpi). Heat maps of control gene RPKM values (log ${ }_{10}$ ) from individual PHH donors or different Huh-7.5 cell passages are shown. IRGs, interferon-regulated genes.

\section{RESULTS}

Experimental design and data visualization. Explanted adult $\mathrm{PHH}$ from seven donors (donor 1 [D1] to D7) were plated and checked for viability prior to infection experiments. $\mathrm{PHH}$ s were incubated with conditioned medium (CM) or infected with replication-competent HCV (strain Jc1). Additionally, we inoculated PHHs with UV-inactivated HCV (HCVUv) where sufficient viable patient material was available (D4 to -7). Cellular RNAs were isolated at two time points ( $6 \mathrm{hpi}$ and $72 \mathrm{hpi}$ ) to enable monitoring of global hepatocyte transcriptional changes by RNA-seq and quantification of HCV RNA (Fig. 1A). Infection experiments in Huh-7.5 cells were also performed in parallel.

After mapping RNA-seq data to the hg38 genome scaffold, raw count data were normalized (reads per kilobase of transcript per million mapped reads [RPKM]) to allow comparison of global gene expression profiles within and between experiments. Mean RPKM values for all expressed genes were plotted for CM-treated PHHs versus HCV- or $\mathrm{HCV}^{\mathrm{UV}}$-infected PHHs, and correlation analyses were performed at both 6 hpi and 72 hpi (Fig. 1B). These analyses revealed that all comparisons were highly significant $(P<0.0001)$, with Pearson's $r$ correlation close to 1 , indicating that the majority of 
hepatocyte mRNAs are expressed at steady state and not significantly dysregulated upon HCV infection. Of note, Pearson's $r$ was lowest at 72 hpi with replication-competent HCV (0.9566), indicating greater numbers of dysregulated hepatocyte genes under these conditions (Fig. 1B). Principal-component analyses (PCAs) were performed separately on samples from individual donors. While differences were observed between donors, separation of HCV-infected PHHs from CM/HCV Uv-treated cells and temporally distinct clusters at 6 and $72 \mathrm{~h}$ were generally observed (see an example plot from D4 in Fig. 1C). These analyses validate our approach of using time-matched infected and uninfected $\mathrm{PHH}$ to avoid mixing of signals associated with HCV infection versus gradual hepatocyte dedifferentiation upon plating.

HCV infection activates antiviral defenses in PHHs but not Huh-7.5 cells. The transcript abundances of a panel of selected control genes were compared in $\mathrm{PHHs}$ and highly permissive Huh-7.5 cells (21), both with and without HCV infection. Comparable abundant expression of the hepatocyte marker $A L B$ and transcripts encoding HCV entry and replication cofactors was detected in both $\mathrm{PHH}$ s and Huh-7.5 cells. Their expression remained stable across the experimental time course, suggesting no reduction in HCV permissiveness and no modulation of expression due to infection (Fig. 1D). As expected, the expression of lung-specific transcripts was either minimal or absent.

The baseline expression of a panel of IFN-regulated genes (IRGs) was detectable in $\mathrm{PHHs}$, which was not further boosted upon infection with HCVUv at either 6 or $72 \mathrm{hpi}$. While minimal IRG upregulation was observed upon infection with replication-competent HCV at $6 \mathrm{~h}$, substantial induction was observed at $72 \mathrm{hpi}$. In contrast, the basal IRG expression level was demonstrably low or completely absent in Huh-7.5 cells compared to $\mathrm{PHHs}$, and no induction was observed upon HCV infection at either sampling point.

Antiviral defenses in PHHs suppress HCV replication and completely abrogate virion release. To validate our RNA-seq data, we performed RT-qPCR on a selected set of test genes and compared the levels of gene induction in both systems. These data confirm a remarkable level of concordance between the two systems and confirm that our RNA-seq data accurately record both steady-state and virus-inducible gene expression (Fig. 2A). Due to the limitations of working with explanted hepatocytes, which include intact innate immune suppression of viral replication and limited sample material per donor, the percentage of infected $\mathrm{PHH}$ could not be accurately determined. To investigate the effects of IRG induction on HCV RNA replication, viral RNA (vRNA) RT-qPCR was performed on cellular RNAs from both PHHs and Huh-7.5 cells (Fig. 2B). For replication-competent HCV, genome copy numbers did not increase between 6 and 72 hpi in $\mathrm{PHHs}$, consistent with innate immune suppression of viral replication (Fig. 2B, top). In contrast, a 2-log increase in vRNA was apparent in Huh-7.5 cells over the same time course, consistent with a lack of IRG upregulation and unimpeded viral replication (Fig. 2B, bottom). As expected, minimal signal was detected in CM- or $\mathrm{HCV}^{\mathrm{UV}}$-treated cells. To determine the rates of infectious particle production, $50 \%$ tissue culture infective dose $\left(\mathrm{TCID}_{50}\right)$ titrations were performed in parallel on supernatants harvested from infected PHHs and Huh-7.5 cells (Fig. 2C). Titers at 6 hpi likely represent carryover of the initial inoculum despite extensive washing. While infectious virion secretion was completely absent in supernatants from $\mathrm{PHHs}$ at $72 \mathrm{hpi}$, HCV virions were detected at $\sim 1 \times 10^{5} \mathrm{TCID}_{50} / \mathrm{ml}$ in supernatants from Huh-7.5 cells at $72 \mathrm{hpi}$. To investigate this further, virion secretion was determined in $\mathrm{PHH}$ that were pretreated with the JAK/STAT inhibitor ruxolitinib prior to infection. Ruxolitinib pretreatment rescued HCV virion release at $72 \mathrm{hpi}$, indicating that the ablation of virion production in PHHs is mediated by JAK/STAT-inducible immunity (Fig. 2C, top). Moreover, these results were confirmed via immunofluorescence staining for viral antigen. At 72 hpi, perinuclear NS5A localization in PHHs was visible only after JAK/STAT inhibition (Fig. 2D) but readily detected in Huh-7.5 cells without pharmacological immune suppression (Fig. 2E). Together, these data indicate that plated $\mathrm{PHH}$ possess intact innate 
A
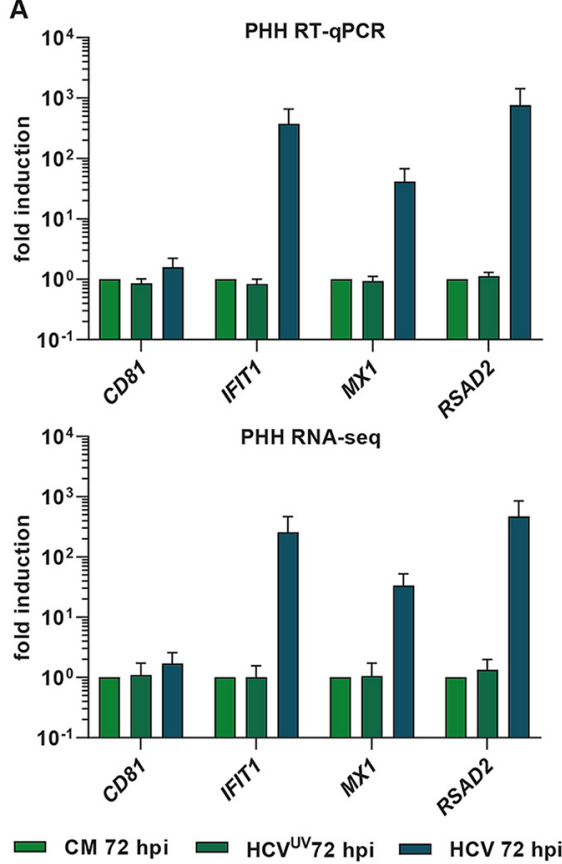

D

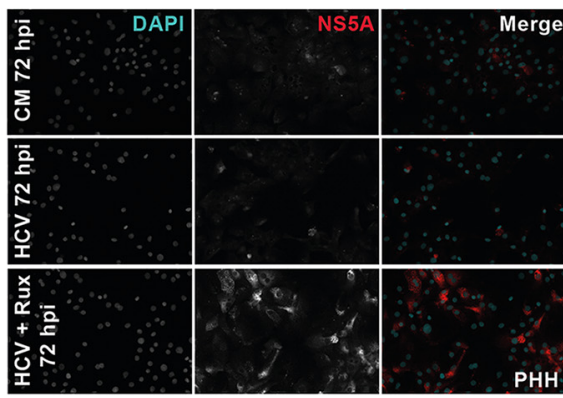

B
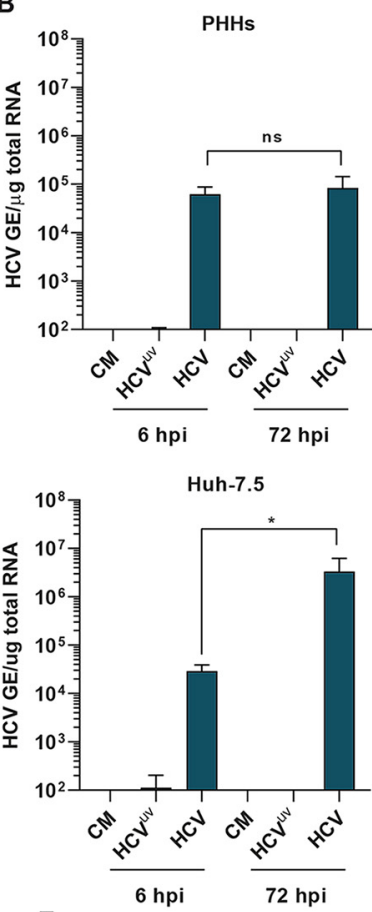

E

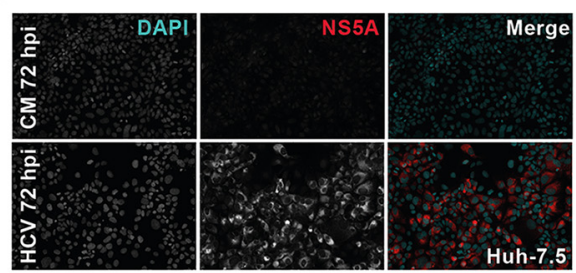

C
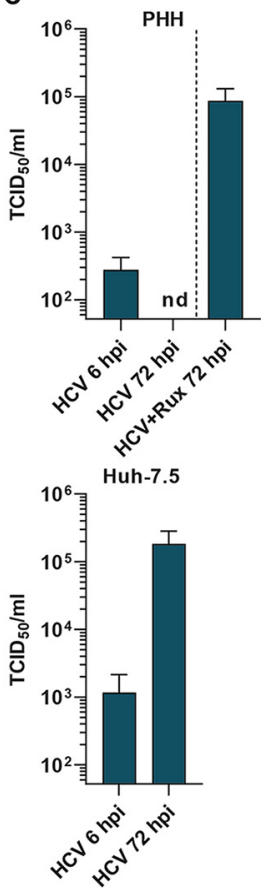

Huh-7.5

FIG 2 Intact innate immunity suppresses HCV propagation in PHHs. (A) Comparative gene induction of four control genes measured by RT-qPCR or RNA-seq ( $n=4$ donors). (Top) Fold gene induction determined by RTqPCR and calculated by the $2^{-\Delta \Delta C T}$ method (43) compared to CM-treated PHHs. (Bottom) Fold gene induction based on RNA-seq data for the same samples under identical conditions. (B) Intracellular HCV RNA copies in HCV-infected PHHs ( $n=4$ donors) (top) and Huh-7.5 cells $(n=3)$ (bottom). Bars represent HCV RNA GE (genome equivalents) per $1 \mu \mathrm{g}$ total RNA. ${ }^{*}, P$ value of $<0.05$; ns, not significant. Data were log transformed, and a onetailed, unpaired $t$ test was applied to each comparison. (C) Secretion of infectious virions in supernatants of HCV-infected PHHs (top) and Huh-7.5 cells (bottom). Numbers of infectious particles in PHH supernatants at 72 hpi were under the LOQ of the assay at a 1:3 dilution. The dashed line indicates a separate experiment with PHHs from four additional donors, preincubated with $10 \mu \mathrm{M}$ ruxolitinib (Rux), a JAK/STAT inhibitor, prior to and directly after HCV infection. (D and E) Immunofluorescence staining of viral NS5A protein in PHHs (D) or Huh7.5 cells (E). PHHs were treated with CM or infected with HCV for $72 \mathrm{~h}$ with or without ruxolitinib pretreatment. NS5A staining is shown in red, with nuclear counterstaining (4',6-diamidino-2-phenylindole [DAPI]) in blue.

immunity, which suppresses initial HCV replication and completely blocks virion release. This inducible immunity is absent in Huh-7.5 cells.

$\mathrm{HCV}$ infection of PHHs dysregulates gene programs associated with diverse biological functions. Statistical analyses were performed to quantify differentially expressed genes (DEGs) induced upon HCV infection of PHHs (false discovery rate [FDR] $P$ value of $<0.05$ ). For replication-competent $\mathrm{HCV}$ infections, a temporally structured increase in DEGs was observed (Fig. 3A, left, and Fig. 3B), which partially overlapped (Fig. 3C). At 6 hpi, 81 infection-induced DEGs were apparent; this number was markedly amplified at $72 \mathrm{hpi}$ (DEGs, $n=2,985$ ) and indicates that HCV infection ultimately induces a substantial shift in the PHH transcriptional landscape (Fig. 3A, left, and Fig. $3 \mathrm{~B}$ ). For HCVuv infections, a contrasting temporally structured reduction in 


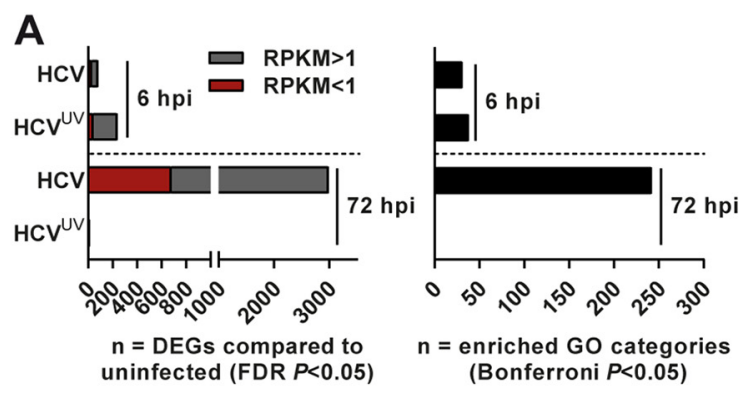

B DEGs PHH Ghpi infected with HCV
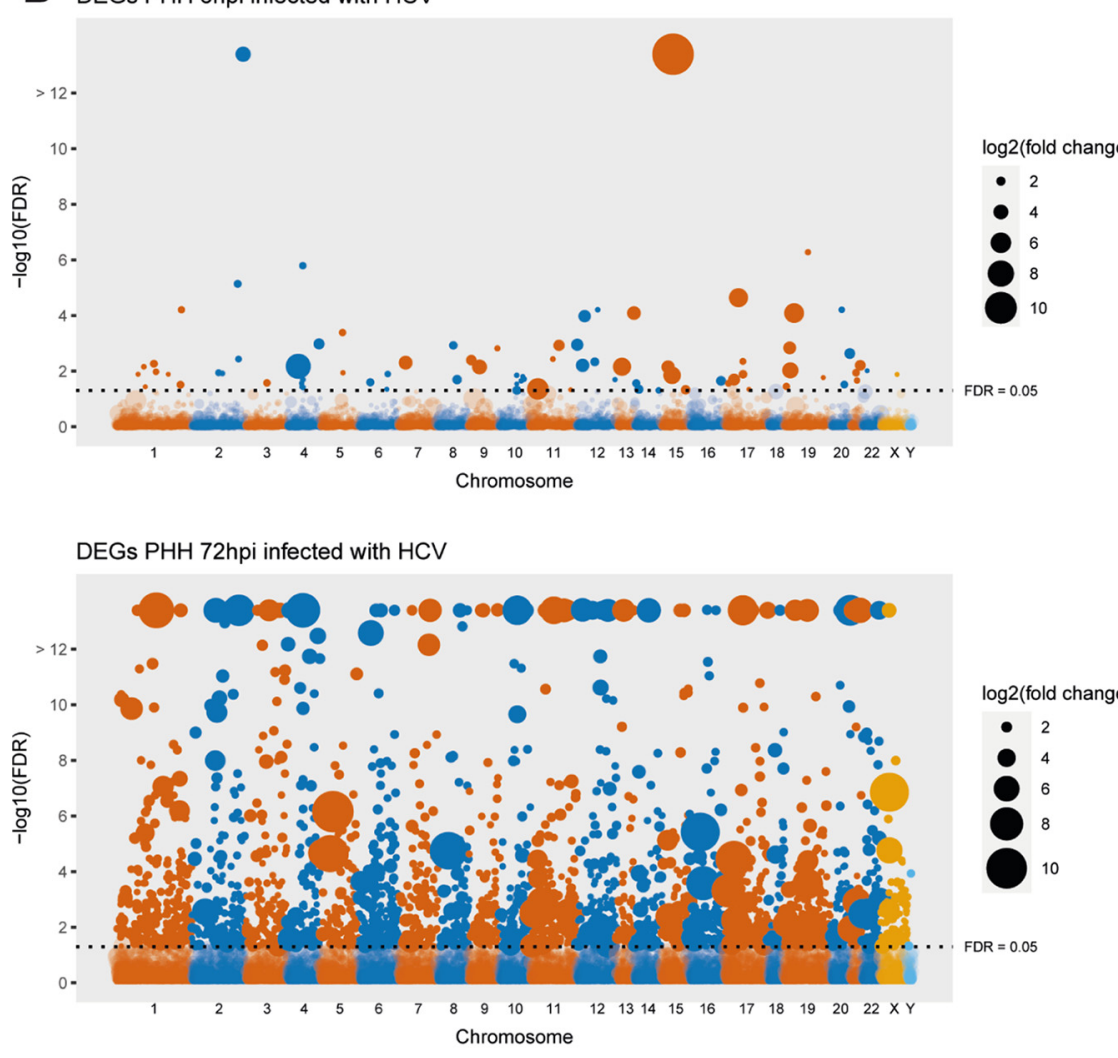

E Uninfected
PHH HCV6 hpi
Uninfected
PHH HCV72 hpi

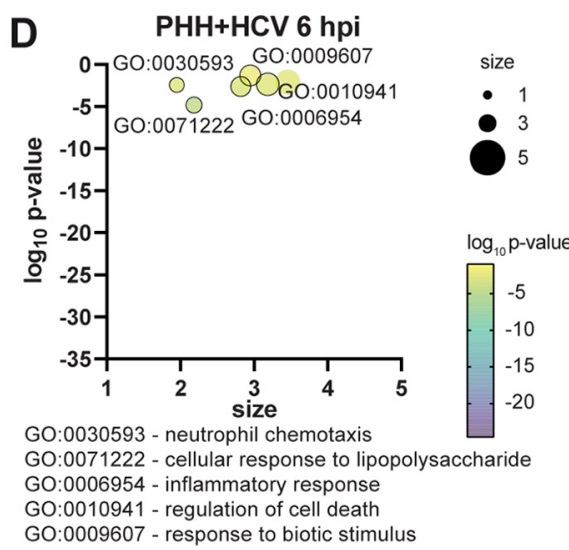

$\mathrm{PHH}+\mathrm{HCV}^{\mathrm{uV}} 6 \mathrm{hpi}$

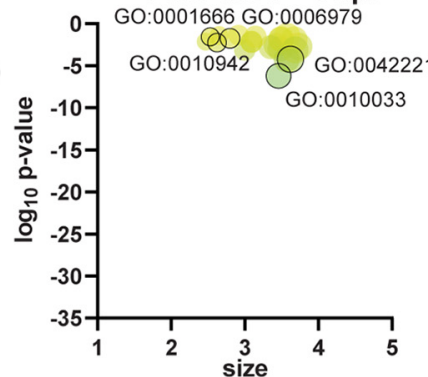

GO:0001666 - response to hypoxia GO.0010942 - positive regulation of cell death GO:0006979 - response to oxidative stress GO:0042221 - response to chemical

GO:0010033 - response to organic substance

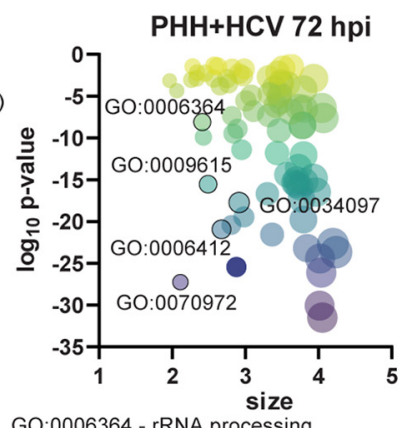

GO:0006364 - rRNA processing

GO:0009615 - response to virus

GO:0034097 - response to cy

GO:0070972 - protein localization to endoplasmic reticulum

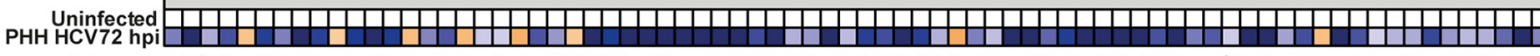

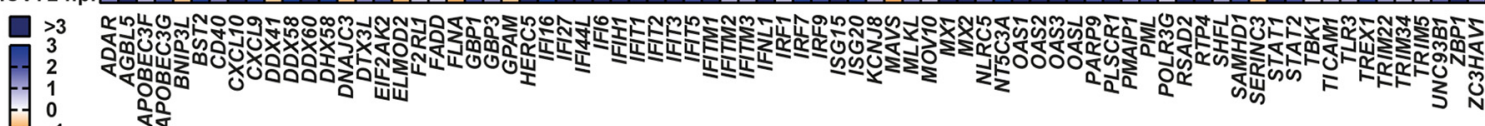
$\log _{2} \mathrm{FC}$

PHH HCV 72 hpi - (GO:0045047) Protein targeting to ER (n=81) РНн Uninfected Uninfected

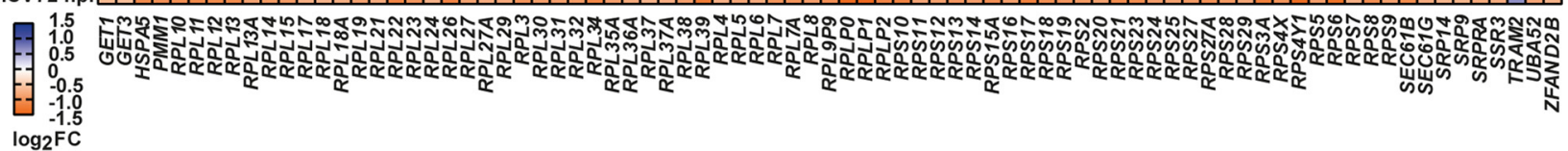

FIG 3 HCV infection induces temporally structured and functionally diverse gene programs in PHHs. (A) Comparison of the numbers of HCV- and HCV ${ }^{\mathrm{U}}$-induced DEGs (left) and enriched GO categories (right) at 6 and 72 hpi. (B) Genomic location of HCV-induced transcripts. Manhattan plots compare transcript abundances in uninfected versus HCV-infected PHHs at 6 hpi (top) and 72 hpi (bottom). Circles represent individual gene comparisons, with sizes proportional to average RPKM fold 
DEG signatures was observed: an initial transcriptional response to HCV ${ }^{\mathrm{Uv}}$ was detected at $6 \mathrm{hpi}(n=239)$, which declined to virtually no detectable dysregulation at $72 \mathrm{hpi}$ $(n=12)$ (Fig. 3A, left).

To determine the biological processes associated with HCV-induced DEGs, gene ontology (GO) enrichment analyses were performed. At 6 hpi, HCV- and HCVUV-enriched GO categories were associated with shared and distinct biological processes (Fig. 3A, right, and Fig. 3D, top two panels). Of note, only PHHs infected with replication-competent HCV demonstrated enrichment of GO categories associated with IFN signaling or pathogen defense: dysregulation of these gene classes was absent in HCVuv-infected $\mathrm{PHH}$. These data represent the early yet restricted signatures of the hepatocyte antiviral response and confirm that this response is induced only by replication-competent vRNAs.

Proportional to the quantity of HCV-induced DEGs, a large number of highly significantly enriched GO categories were identified at $72 \mathrm{hpi}$, which were associated with diverse biological processes (Fig. 3A, right, and Fig. 3D, bottom). The overlap with DEGs representing described IRGs $(n=390)(7,22)$ or IRG interactors $(n=2,582)(22)$ (Fig. 3C, bottom) resulted in the enrichment of multiple GO categories associated with antiviral responses and innate immunity (Fig. 3D, bottom). However, many DEGs were not classical IRGs/IRG interactors and represent biological processes not automatically associated with the antiviral response (e.g., ribosome biogenesis, translation initiation, and protein targeting to the ER) (Fig. 3D, bottom). Examples of opposing patterns of gene dysregulation can be seen in Fig. $3 \mathrm{E}$ for two representative $\mathrm{GO}$ categories: while genes associated with defense response to virus are generally strongly upregulated at $72 \mathrm{hpi}$, genes associated with protein targeting to the ER are almost exclusively downregulated. Together, these early snapshots of virus-induced cellular changes confirm temporally regulated transcriptional responses to $\mathrm{HCV}$ infection in adult $\mathrm{PHHs}$, with the magnitude of gene induction increasing exponentially from 6 to $72 \mathrm{hpi}$.

Divergent transcriptional responses to HCV infection and ectopic IRF1 expression in Huh-7.5 cells. Huh-7.5 cells represent the most frequently used cell line for HCV propagation in research. Consequently, we also cataloged global HCV-induced transcriptional responses in these cells for comparative purposes. Similar to PHHs, statistical analyses identified a time-dependent increase in significant HCV-induced DEGs and enriched GO categories (Fig. 4A).

Intrinsic IRF1 expression has been reported to maintain the baseline transcription of a program of antiviral genes, independently of the IFN system $(23,24)$. To quantify the IRF1 regulon in human cells of hepatic origin, we transcriptionally reprogrammed Huh7.5 cells by ectopically expressing IRF1. DEGs and GO enriched categories induced by $\mathrm{HCV}$ at 72 hpi and ectopic IRF1 expression were numerically similar (Fig. 4A and B). However, the underlying dysregulated genes exhibited an $\sim 10 \%$ overlap (Fig. 4 C, top), and the significantly enriched biological processes associated with the dysregulated genes were highly divergent (Fig. 4D). HCV-induced genes in Huh-7.5 cells were associated with diverse biological processes, including the circadian rhythm or amino acid transport (Fig. 4D, top), and showed minimal overlap with HCV-induced genes in PHHs (Fig. 4C, bottom). In contrast, IRF1-regulated genes were largely but not exclusively associated with GO categories related to innate immunity or pathogen defense (Fig. $4 \mathrm{D}$, bottom). Examples of IRF1-mediated gene dysregulation are shown for two representative $\mathrm{GO}$ categories associated with innate immunity (Fig. 4E). In summary, these data expand the IRF1 regulon identified by gene microarrays previously (7) ( $n=130$ ) by an additional 329 genes $(n=459)$ and confirm that Huh-7.5 cells retain the capacity to

FIG 3 Legend (Continued)

changes $\left(\log _{2}\right)$. (C) Number of HCV-induced genes in PHHs at 6 and 72 hpi. The top Venn diagram shows the overlap between DEGs at 6 and 72 hpi, while the bottom diagram shows the gene overlap at 72 hpi with characterized IRGs $\left(^{*}\right)(7)$ and IRG interactors $\left(^{* *}\right)(22)$. Only DEGs with FDR $P$ values of $<0.05$ and RPKM values of $>1$ were included. (D) GO enrichment analysis of HCV-induced DEGs in PHHs. Each circle represents a GO category and is plotted depending on its $P$ value and size. The size illustrates the frequency of the GO term in the underlying GO annotation (46). Representative GO categories are highlighted in graphs, and their full annotation is displayed below each plot. (E) HCV-induced transcripts at $72 \mathrm{hpi}$. Mean ( $n=7$ donors) fold changes (FC) (log 2 ) of HCV-induced DEGs associated with the defense response to virus (top) or protein targeting to the ER (bottom) are shown. 
A

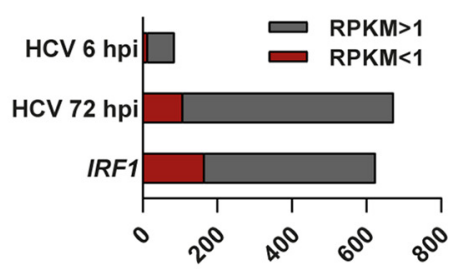

$\mathrm{n}=$ DEGs compared to uninfected (FDR $P<0.05$ )
C

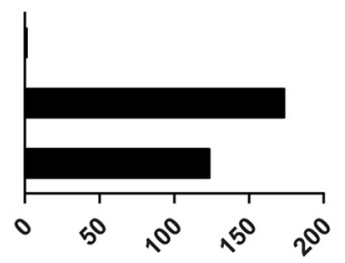

$\mathrm{n}=$ enriched $\mathrm{GO}$ categories (Bonferroni $P<0.05$ )

B

DEGs Huh-7.5 $72 \mathrm{hpi} \mathrm{infected} \mathrm{with} \mathrm{HCV}$
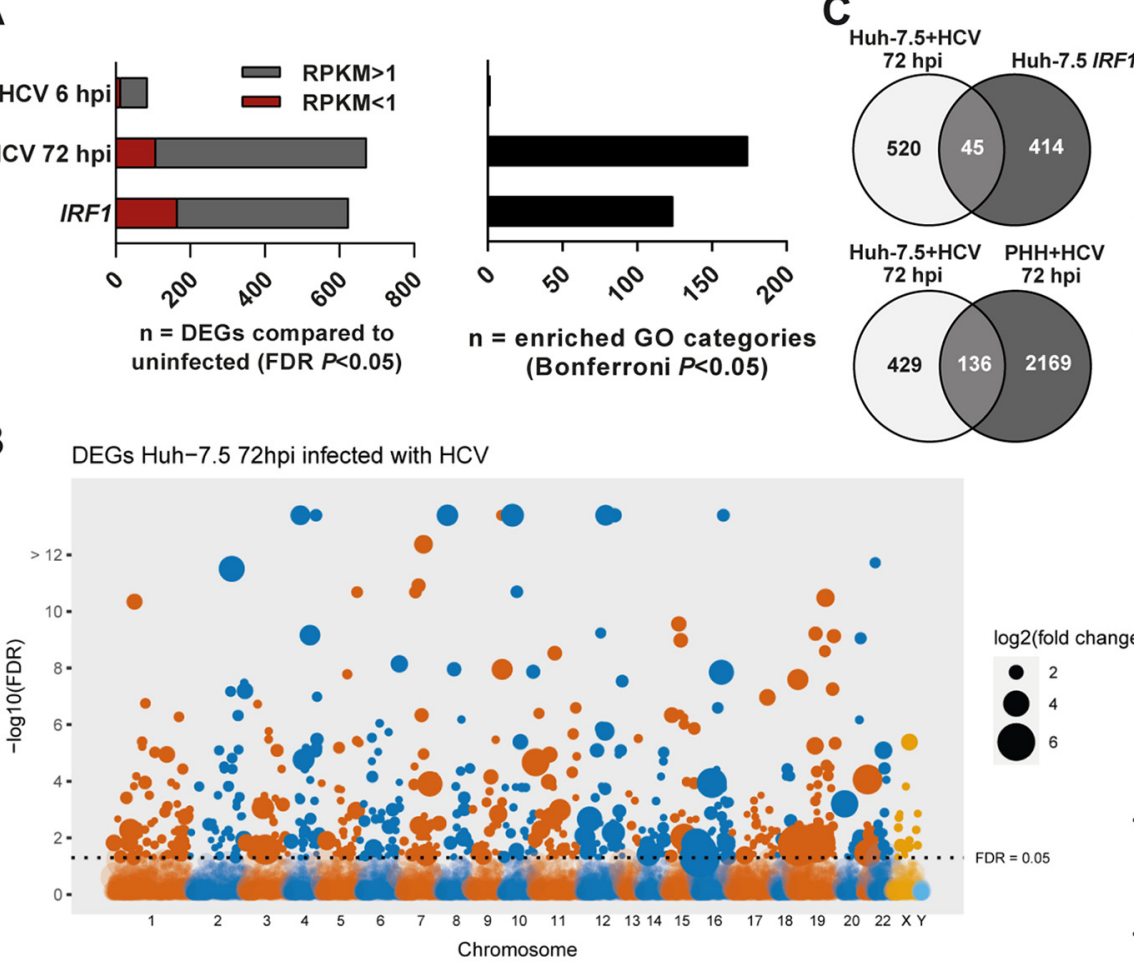

DEGs IRF1 overexpressing cells compared to empty transduced

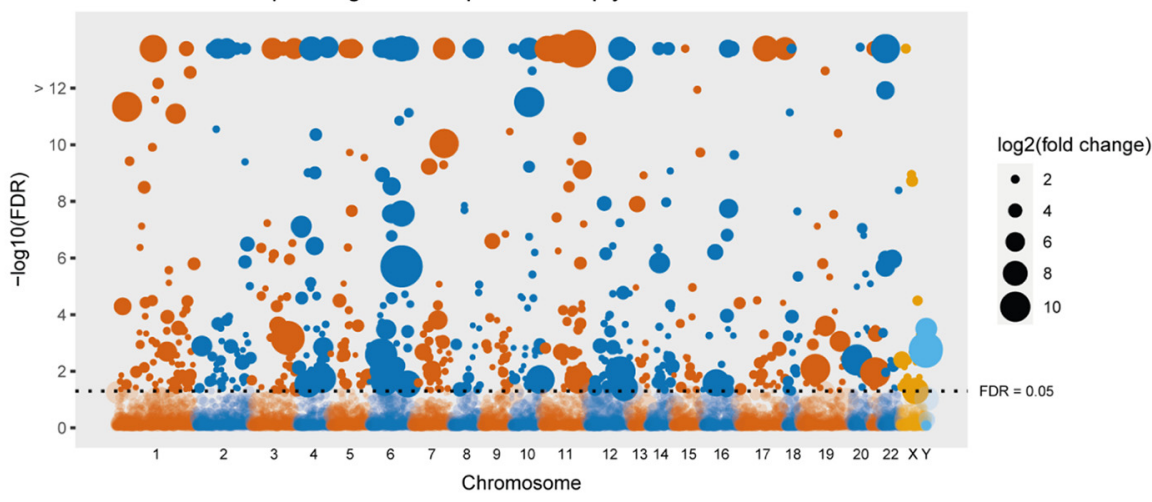

$\log 2$ (fold change)

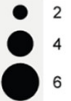

FDR $=0.05$

$$
\text { - }
$$

GO.0000084 - mitotic S phase

GO:0002697 - regulation of immune effector process GO:0034097 - response to cytokine

GO:0098542 - defense response to other organism GO:0002376 - immune system process

\section{E}

Huh-7.5 IRF1 (GO:0045087) Innate immune response $(n=77)$

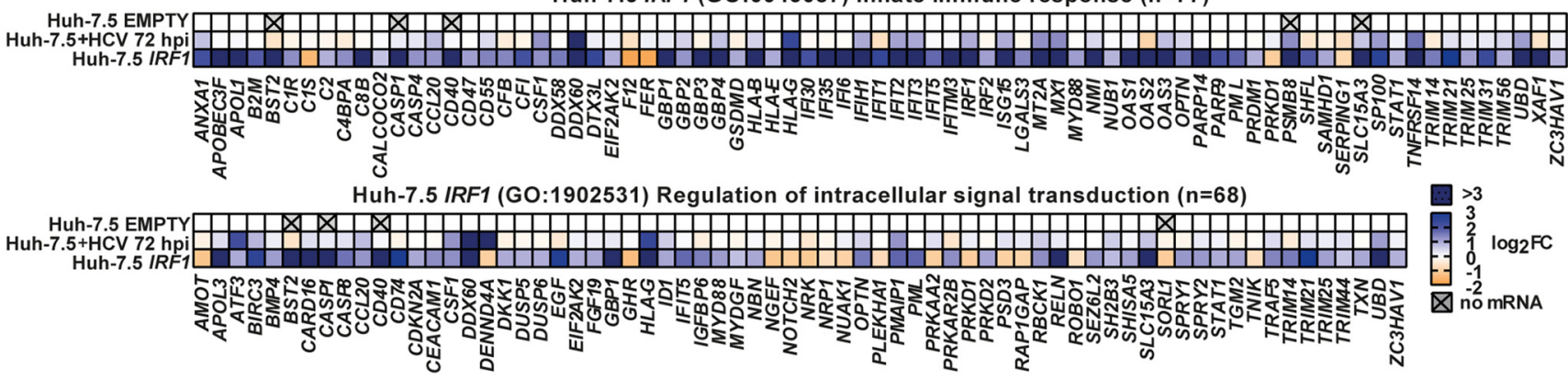

FIG 4 Distinct IRF1- and HCV-mediated transcriptional programs in Huh-7.5 cells. (A) Comparison of numbers DEGs (left) and enriched GO categories (right) upon HCV infection or ectopic IRF1 expression in Huh-7.5 cells. (B) Genomic location of HCV-induced (top) or IRF1-induced (bottom) transcripts in Huh-7.5 cells. Manhattan plots compare the transcript abundances in uninfected Huh-7.5 cells to those in HCV-infected or IRF1-expressing cells. Circles represent individual gene comparisons, with sizes proportional to average RPKM fold changes $\left(\log _{2}\right)$. (C) HCV- and IRF1-induced DEGs show limited overlap. The top Venn diagram shows the overlap between HCV- and IRF1-induced DEGs, while the bottom diagram shows the overlap of HCV-induced DEGs in Huh-7.5 versus PHHs. Only DEGs with FDR $P$ values of $<0.05$ and RPKM values of $>1$ were included. (D) GO enrichment analyses of HCV- and IRF1-induced DEGs in Huh-7.5 cells. Each circle represents a GO category and is plotted depending on its $P$ value and size. The size illustrates the frequency of the $\mathrm{GO}$ term in the underlying GO annotation (46). Representative GO categories are highlighted in graphs, and their full annotation is displayed below each plot. (E) IRF1-induced transcripts in Huh-7.5 cells. Mean fold changes (log 2 ) of IRF1induced DEGs associated with the innate immune response (top) or regulation of intracellular signal transduction (bottom) are shown. 
A

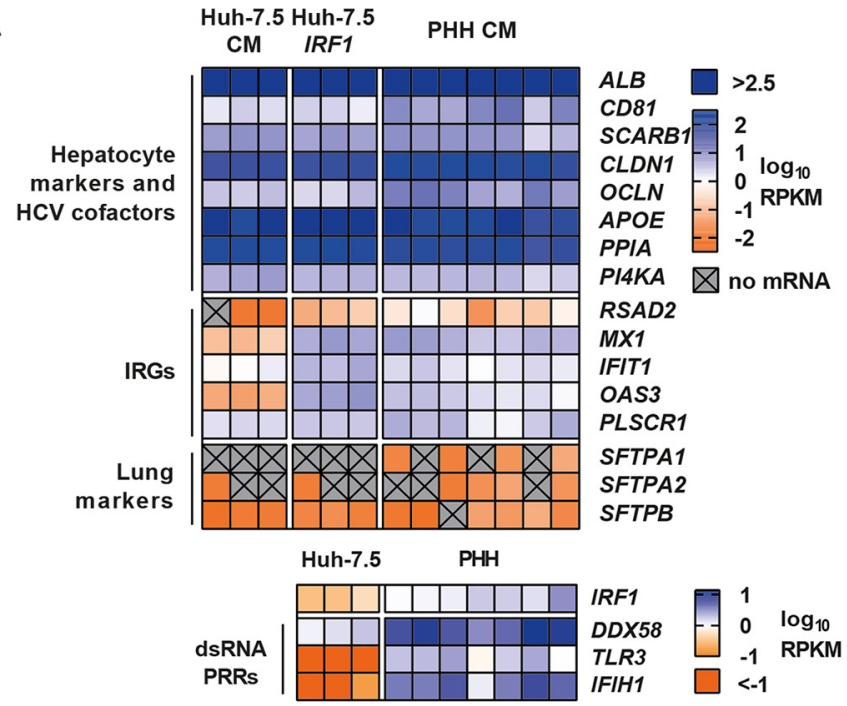

B

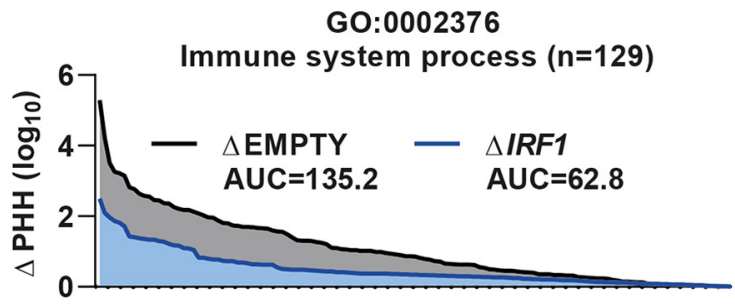

GO:0019222

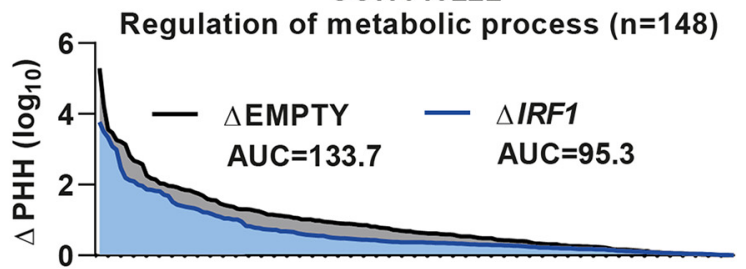

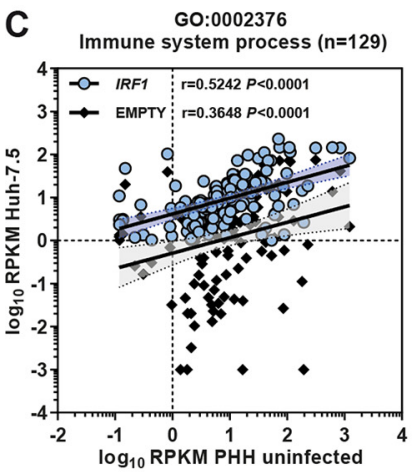
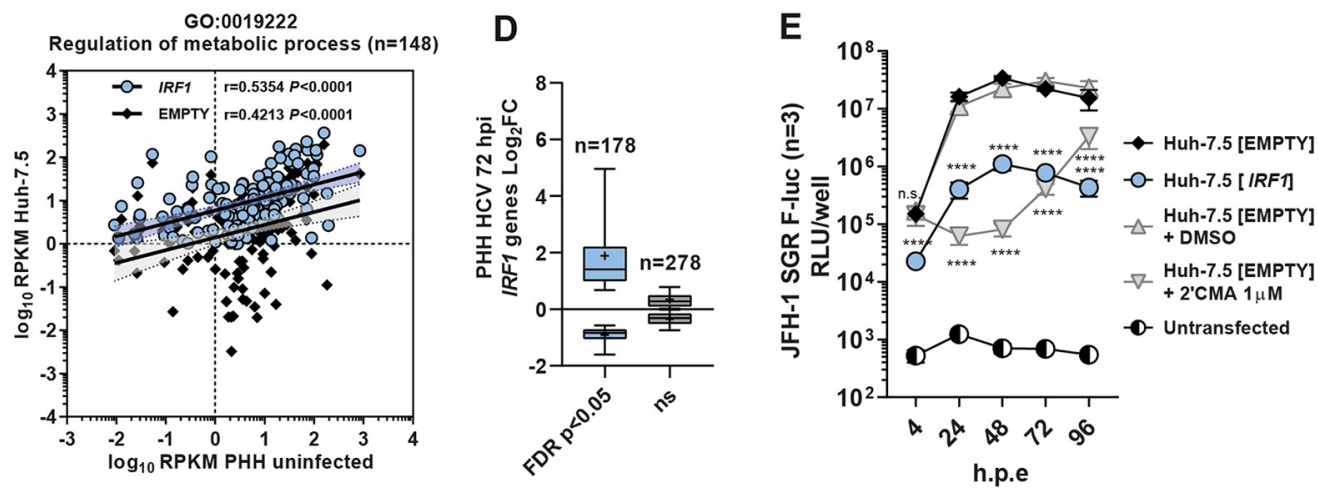

FIG 5 Ectopic IRF1 expression induces an antiviral gene signature in Huh-7.5 cells similar to baseline expression in PHHs. (A) Baseline expression of IRGs and IRF1. (Top) Basal IRG expression in Huh-7.5 cells with and without IRF1 expression, compared to uninfected PHHs. (Bottom) Comparison of baseline expression of IRF1 and pattern recognition receptors (PRRs) that recognize double-stranded RNA (dsRNA) in Huh-7.5 cells and PHHs. (B) Area under the curve (AUC) analysis. Shown are differences in IRF1-regulated gene expression levels for two distinct GO categories from Huh-7.5 EMPTY and Huh-7.5 IRF1 cells, compared to expression in PHHs. Higher AUC values indicate more divergent expression profiles. (C) Correlation plots of IRF1-regulated genes from the same two GO categories. RPKM values are plotted for individual genes, and simultaneous comparison of their expression in uninfected PHHs to those in both Huh-7.5 EMPTY and Huh-7.5 IRF1 cells is visualized. Pearson's $r$ and $P$ values for each comparison are inset. (D) HCV-inducible IRF1-regulated genes in PHHs at $72 \mathrm{hpi}$. +, mean $\log _{2}$ fold change in expression. (E) Restriction of HCV replication and translation by ectopic IRF1 expression. Huh-7.5 EMPTY and Huh-7.5 IRF1 cells were electroporated with a subgenomic replicon of JFH-1. h.p.e, hours postelectroporation. ${ }^{* * * *}, P<0.0001$. Data were log transformed, and multiple unpaired $t$ tests were performed comparing Huh-7.5 EMPTY versus Huh-7.5 IRF1 cells and Huh-7.5 EMPTY cells with DMSO versus Huh-7.5 EMPTY cells with 2'CMA, respectively.

mount antiviral defenses. Our highly sensitive RNA-seq profiling identified 271 additional low-abundance transcripts as significantly dysregulated (FDR $P$ values of $<0.05$; final RPKM value of $<1$ ), although these were omitted from subsequent analyses to increase stringency.

Baseline IRF1 expression coordinates an intrinsic antiviral program in PHHs. Using our previously selected panel of control genes (Fig. 1D), we visualized the baseline expression of IRGs in uninfected Huh-7.5 cells transduced with an empty lentivirus (Huh-7.5 EMPTY), Huh-7.5 IRF1 cells, and uninfected PHHs. We observed highly similar baseline IRG expression levels in Huh-7.5 IRF1 cells and uninfected PHHs, distinct from that in Huh-7.5 EMPTY cells (Fig. 5A, top). We also visualized the baseline expression of IRF1 in Huh-7.5 cells and PHHs and compared the expression levels to those of pattern recognition receptors (PRRs) that recognize cytosolic double-stranded RNA (dsRNA) (Fig. 5A, bottom). These data confirm minimal IRF1 expression in Huh-7.5 cells, 4-fold lower than PHH baseline expression levels. These observations further reveal much higher baseline expression levels of the cytosolic dsRNA sensors TLR3, DDX58 (RIG-I), and IFIHI (MDA5) in PHHs than in Huh-7.5 cells. 
Next, we compared the mean expression levels of IRF1-regulated genes from both Huh-7.5 EMPTY and Huh-7.5 IRF1 cells to the expression levels in PHHs. Applying robust statistical methods to compare gene subsets from two distinct enriched GO categories within the IRF1 regulon, we performed area under the curve (AUC) and correlation analyses. AUC analyses confirmed that the expression of IRF1-regulated genes in Huh-7.5 IRF1 cells was more closely related to baseline expression in PHHs than in Huh-7.5 EMPTY cells (Fig. 5B). Of note, this pattern was more pronounced for genes associated with immune system processes (GO:0002376) than for genes associated with the regulation of metabolic processes (GO:0019222). Correlation analyses compared the mean expression levels of individual IRF1-regulated genes in PHHs to those in both Huh-7.5 EMPTY and Huh-7.5 IRF1 cells (Fig. 5C). While all comparisons were significantly correlated $(P<0.0001)$, higher Pearson's $r$ values were observed for Huh-7.5 IRF1 cells ( $r=0.52$ and $r=0.53$ ) than for Huh-7.5 EMPTY cells ( $r=0.36$ and $r=0.42)$, indicating that ectopic expression of IRF1 pushes the Huh-7.5 cell transcriptome to a more $\mathrm{PHH}$-like state. Correspondingly, this pattern was again more pronounced for immune system process genes (GO:0002376) than for genes associated with the regulation of metabolic processes (GO:0019222) (Fig. 5C). IRF1 also represents an IRG, and its expression is further boosted by HCV infection (Fig. 3E, top). Consequently, we next determined what proportion of the IRF1 regulon is further dysregulated upon HCV infection of PHHs (Fig. 5D). These analyses identify 178 IRF1-regulated genes whose expression is significantly dysregulated by HCV infection of PHHs, indicating that IRF1 also contributes to inducible immunity.

To determine the effect of ectopic IRF1 expression on HCV RNA replication, we transfected either Huh-7.5 EMPTY or Huh-7.5 IRF1 cells with a subgenomic replicon (SGR) containing the nonstructural proteins NS3 to NS5B from strain JFH-1 coupled to a firefly luciferase (F-luc) reporter and monitored luciferase accumulation over time (Fig. 5E). These experiments confirm that the transcriptional program mediated by IRF1 has the ability to reduce HCV RNA replication significantly. We also performed transfections in the presence of the HCV replication inhibitor 2' C-methyl adenosine (2'CMA) or a dimethyl sulfoxide (DMSO) vehicle control. The significant IRF1-mediated reduction in relative luciferase units (RLU) was also apparent at $4 \mathrm{~h}$ posttransfection in Huh7.5 IRF1 cells, which was not the case in $2^{\prime}$ CMA-treated cells, indicating that the IRF1 gene program also negatively impacts viral genome translation, possibly due to direct targeting or competition with host IRG mRNAs for available ribosomes (Fig. 5E). In summary, these analyses provide supportive evidence that IRF1 coordinates the baseline expression of antiviral effector genes in PHHs in the absence of infection and also contributes to inducible immunity. The antiviral effect mediated by the IRF1 gene program was able to reduce HCV replication/translation by 1 to 2 logs. Thus, in addition to the observed triggering of antiviral defenses by HCV, it is likely that this suite of intrinsically expressed genes in $\mathrm{PHH}$ contributes to the defense against incoming virus.

Comparison of transcriptional regulators and targeted canonical pathways. To determine upstream transcriptional regulators that orchestrate changes in gene expression induced by HCV infection of PHHs or Huh-7.5 cells or ectopic IRF1 expression in Huh-7.5 cells, we performed ingenuity upstream regulator analysis (Qiagen). Focusing on nuclear transcription factors (TFs), we observed significant activation (Fig. 6A, blue) or inhibition (orange) $(P<0.05)$ of diverse transcriptional regulators, with distinct and overlapping TF profiles observed for each data set. Limited overlap $(n=5)$ was observed between the TFs of coordinating transcriptional responses to HCV infection in PHHs and Huh-7.5 cells. Of note, low oxygen tension has been demonstrated to enhance HCV RNA replication (25), and activation of hypoxia-inducible factor 1 alpha (HIF1A) is observed in both systems, which is a known regulator of transcriptional responses to hypoxia (26).

As expected, considerable overlap was observed between the transcriptional regulators coordinating the observed patterns of gene dysregulation detected in HCVinfected PHHs and IRF1-reprogrammed Huh-7.5 cells, as IRF1 is further upregulated upon HCV infection of PHHs. Multiple shared TFs known to activate antiviral programs 
A

PHH HCV 72 hpi

Huh-7.5 IRFI

Huh-7.5 HCV 72 hpi

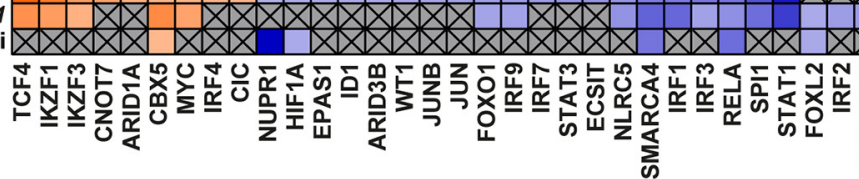
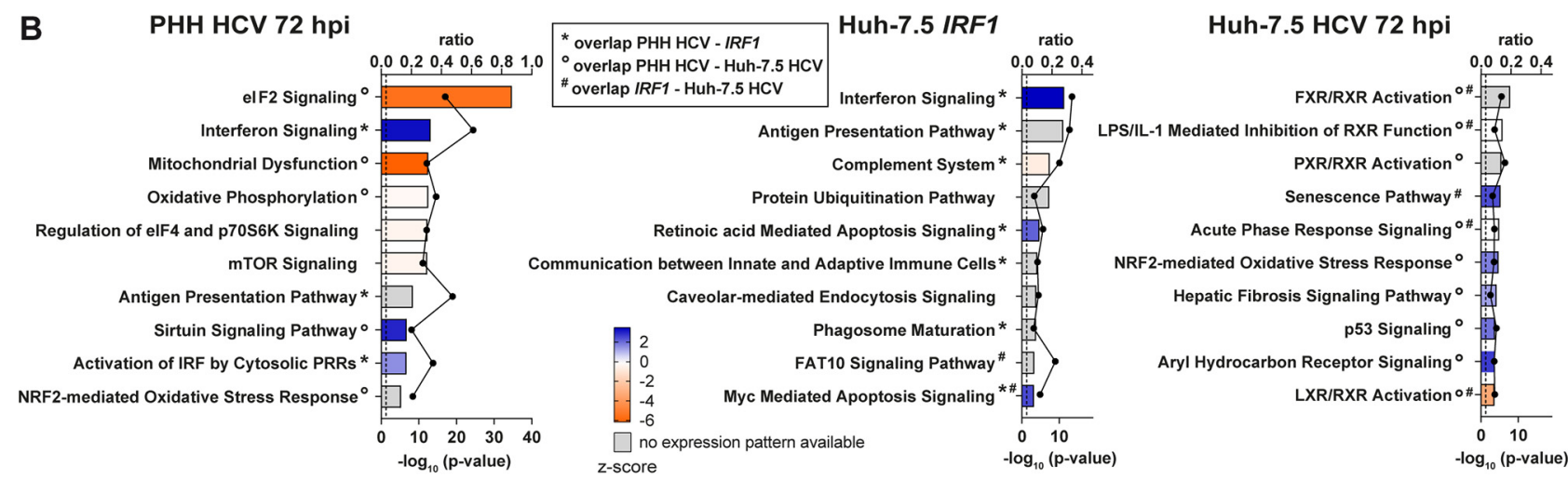

C
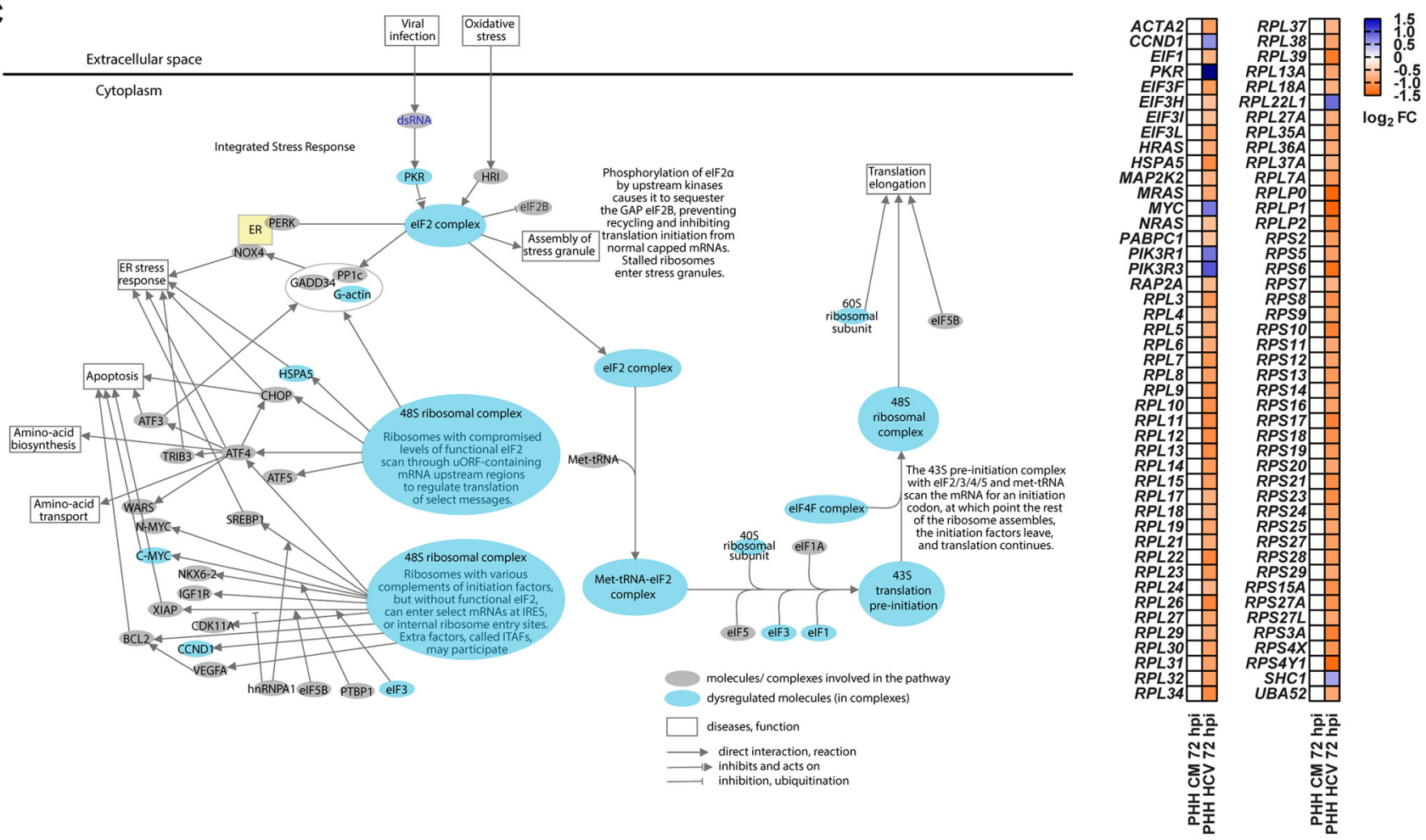

$\log _{2}$ FC

FIG 6 Upstream transcriptional regulators and canonical pathways dysregulated by HCV infection or ectopic IRF1 expression. (A) Upstream transcriptional regulators controlling DEGs. Ingenuity pathway analysis (IPA) was used to calculate upstream regulators based on significant DEGs identified under the three presented conditions. Z-scores indicate activation or inhibition of individual regulators. Displayed are only the transcriptional regulators with a $P$ value of $<0.05$ and a Z-score higher than 2 or lower than -2. (B) Canonical pathway analyses of HCV- and IRF1-induced DEGs in PHHs and Huh-7.5 cells, respectively. Pathways are plotted with the corresponding $P$ values (bars) and ratios between dysregulated molecules in our data sets and all molecules belonging to that pathway (linked black circles). The dotted line represents the significance threshold $(P=0.05)$. The bar color represents the expression Z-score. LPS, lipopolysaccharide; IL-1, interleukin-1. (C) Visualization of the top HCV-dysregulated pathway in PHHs at 72 hpi (elF2 signaling) and its underlying DEGs. (Left) Modified cartoon of elF2 signaling as determined by IPA. (Right) Heat map displaying fold changes of significant DEGs involved in this pathway. GAP, GTPase-activating protein; UORF, upstream open reading frame; ITAFs, internal ribosome entry site (IRES)-transacting factors.

were activated. Furthermore, these analyses demonstrate that ectopic IRF1 expression additionally activates an array of transcriptional regulators, resulting in a broad induction of antiviral effectors, and highlight that IRF1 coordinates a complex web of multiple TFs, which collectively contribute to the IRF1 regulon. 
To further explore these data, we used ingenuity pathway analysis (IPA) (Qiagen) to investigate which canonical cellular pathways are affected by HCV- or IRF1-mediated gene dysregulation. Shared and distinct canonical pathways were targeted in all three systems (Fig. 6B [only the top 10 hits are presented]). Notably, for HCV infection of PHHs, a number of targeted pathways were associated with innate immune responses, including "interferon signaling," "antigen presentation pathway," and "activation of cytosolic IRF by PRRs." Targeted transcriptional dysregulation of pathway components associated with IFN-mediated innate immunity was absent in HCV-infected Huh-7.5 cells. As Huh-7.5 cells do not produce IFN and possess impaired antiviral effector responses, we reasoned that these HCV-targeted pathways represent proviral transcriptional manipulation to facilitate HCV propagation. Cell-intrinsic pathways targeted by IRF1 were generally involved in innate immunity and exhibited some crossover with HCV-infected PHHs, including "interferon signaling," "antigen presentation pathway," and "complement system."

In HCV-infected PHHs, in addition to pathways associated with classical IFN-mediated innate immunity, we also observed targeting of unrelated pathways, including "elF2 signaling," "mitochondrial dysfunction," and "mTOR signaling." The top targeted pathway in HCV-infected PHHs was "elF2 signaling," and detailed inspection of this pathway identified significantly dysregulated molecules at multiple pathway stages (Fig. 6B, left). The corresponding changes in gene expression for targeted molecules within the "elF2 signaling" pathway highlight the significant downregulation of genes that comprise the structural components of ribosomes and the translation preinitiation complex. In contrast, significant upregulation of the protein kinase $\mathrm{R}$ gene $(P K R)$ and MYC is observed (Fig. 6B, right). Together, these analyses provide a broad overview of the transcriptional regulators that orchestrate HCV- or IRF1-mediated gene dysregulation and the downstream cell-intrinsic pathways that they target.

\section{DISCUSSION}

In this study, we sought to quantify and dissect initial global transcriptional responses to HCV infection of authentic target cells: adult PHHs. Acquiring these data in vivo is particularly challenging. Indeed, HCV has a highly restricted cellular tropism and efficiently infects only human hepatocytes. The liver is a solid organ that is composed of multiple cell types (27), and while hepatocytes represent the major cell type, these cells are not readily accessible for sampling. Acute HCV infection is also often asymptomatic, so initial infections often go unnoticed. Here, we sought to overcome these hurdles by performing ex vivo infections of $\mathrm{PHH}$ isolated from adult donors who were not previously infected with HCV or treated with IFN. Our quantification of initial acute-phase responses to infection in adult PHHs provides a snapshot of early perturbations in the hepatocyte transcriptional landscape induced by HCV infection.

In agreement with the reported spatiotemporal shifting of early HCV replication complexes within the cytosol, patterns of HCV-induced gene dysregulation were time structured in adult PHHs. Limited gene induction was observed at $6 \mathrm{hpi}$, where initial viral replication complexes are associated with ribosomes (19). However, weak induction of a restricted panel of IRGs was detected, which were not upregulated in $\mathrm{HCV}^{\mathrm{uv}}$-treated cells, and represents the first signatures of the $\mathrm{PHH}$ antiviral response to replication-competent HCV. This limited induction of antiviral effectors shortly after infection could represent HCV NS3/4A-mediated targeting of MAVS, which dampens host antiviral responses (28), and contrasts with IFN or poly $(\mathrm{I} \cdot \mathrm{C})$ treatment of $\mathrm{PHHs}$, where early DEG induction at $6 \mathrm{~h}$ is associated with broad IFN-mediated responses (29). Alternatively, intrinsic IRF1 expression in PHHs may limit the replication capacity of incoming virus (see below for further discussion), reducing the accumulation of dsRNA and therefore delaying broad innate immune induction. In contrast to 6-hpi DEGs, the spatial transitioning of viral replication complexes to remodeled ER membranes at 72 hpi (20) coincided with an exponential amplification of HCV infectionmediated transcriptional dysregulation. We detected significant dysregulation of $\sim 3,000$ genes, associated with a diverse array of biological processes. 
At 72 hpi in PHHs, we observed expansive induction of IFN-triggered antiviral effector genes, promoting the suppression of viral replication and the abrogation of particle release. However, $80 \%$ of HCV-infected individuals fail to mount effective responses, facilitating the progression to chronicity, and viruses have evolved a variety of innate immune evasion strategies to promote their propagation, which includes host translational shutoff (30). Indeed, while HCV infection results in effective IRG induction, virusinduced phosphorylation of PKR inhibits the $\alpha$ subunit of eukaryotic initiation factor 2 (eIF2 $\alpha$ ) and therefore blocks host IRG protein translation (31). PKR activation is therefore advantageous to HCV and prevents clearance because while the translation of capped host mRNAs is dependent on elFs, HCV polyprotein translation occurs independently of elFs using an internal ribosome entry site (IRES) located in the $5^{\prime}$ untranslated region (UTR) of the viral genome (32). Along with the previously described HCV inhibition of elF2 $\alpha$ (31), our transcriptional profiling identifies broad downregulation of multiple components of the host translational machinery. Mechanistically, this process is likely mediated by the TF MYC, which is known to directly regulate ribosome biogenesis and translation, controlling the expression of the RPS and RPL proteins of the small and large ribosomal subunits, in addition to the gene products necessary for rRNA processing, nuclear export of ribosomal subunits, and mRNA translation initiation (33). Interestingly, in PHHs, significant upregulation of MYC mRNA results in the downregulation of the gene products that it controls (Fig. 6C, right). These data may represent a previously unappreciated mechanism whereby the downregulation of constituent components of the translational machinery arrests the translation of antiviral effector genes. In contrast, MYC is predicted to be inhibited in upstream regulator analysis (Fig. 5A). Detailed investigation of the genes involved in the predicted MYC inhibition and the interactions included in the IPA knowledgebase highlights a number of genes involved in IFN signaling based on microarray analysis in plasmacytoid dendritic cells (pDCs) (34). These genes are highly upregulated upon MYC knockdown in $\mathrm{pDCs}$, and the same genes are induced in the antiviral immune response in $\mathrm{PHHs}$ upon HCV infection. Differences between cellular responses in pDCs and PHHs or upon knockdown of MYC compared to HCV infection may result in the upregulation of MYC in our data set. Together, these data simultaneously capture infection-induced transcriptional signatures associated with proviral translational shutoff and antiviral IFN signaling.

Furthermore, we observed transcriptional dysregulation of additional gene programs that have been reported to modulate susceptibility to HCV. We observed targeting of the mTOR signaling pathway, which has been reported as proviral, with the mTOR inhibitor rapamycin targeting HCV replication in vitro and reducing viral RNA levels in patients posttransplantation (35). HCV infection has also been shown to activate mTOR, and pharmacological inhibition of mTOR was shown to suppress HCV virion assembly and release in vitro (36). Of note, significant targeting of multiple pathways associated with nuclear receptor (NR) signaling was detected in both $\mathrm{PHH}$ and Huh-7.5 cells, further confirming that Huh-7.5 cells retain some HCV-inducible programs that are of biological relevance. NR-mediated signaling regulates transcriptional programs that control host metabolic processes and lipid metabolism and are reported to modulate susceptibility to HCV infection in both humans (37) and mice (38).

In addition to HCV activation of pro- and antiviral cascades, we also investigated the contribution of IRF1-mediated intrinsic immunity in PHHs to the control of HCV replication. IRF1 is a TF that participates in IFN induction but also directly induces a subset of IRGs. Additionally, IRF1 represents a potent panviral restriction factor and a key component of the cellular antiviral response (7). Using microarray analysis, Schoggins et al. identified a panel of 130 partially overlapping, IRF1-regulated genes (>3-fold) via lentiviral overexpression in Huh-7 and STAT1-1- fibroblasts (7). More recently, it has been demonstrated that constitutive expression of IRF1 in immortalized $\mathrm{PH} 5 \mathrm{CH} 5$ cells of hepatic origin (24) and BEAS-2B bronchial epithelial cells (23) coordinates intrinsic antiviral protection independently from the IFN system. Ectopic expression of IRF1 in Huh-7.5 cells enabled us to define a greatly expanded IRF1 regulon, and comparative statistical analysis of Huh-7.5 EMPTY, Huh-7.5 IRF1, and uninfected PHH 
transcriptomes provides supportive evidence that baseline immunity in PHHs in the absence of infection is orchestrated by IRF1. Previous studies have shown IRF1 to be a potent restrictor of HCV replication (7). We confirm this observation and also determine that IRF1 can significantly inhibit HCV subgenome translation in Huh-7.5 cells. While intrinsic IRF1 expression in PHHs maintains a suite of genes that can actively suppress HCV translation and replication, which may contribute to the low levels of gene induction at $6 \mathrm{hpi}$, this translational suppression could be partially overcome by the host translational shutoff that we observe at $72 \mathrm{hpi}$.

In summary, the virus-host interactions that determine the susceptibility of human hepatocytes to initial HCV infection and their capacity to support persistent infection are incompletely defined. The high levels of genetic diversity seen between HCV genotypes point to a long period of association for virus and host coevolution (39), and HCV has evolved multiple strategies to hijack the host cell machinery required to facilitate its propagation while at the same time evading host defenses. We observe early concurrent transcriptional dysregulation of gene programs that facilitate viral persistence, balanced against those that promote viral inhibition. This cellular pro- and antiviral antagonism may keep HCV replication levels below a clearance threshold in the initial phase of infection and ultimately facilitate progression to chronicity.

\section{MATERIALS AND METHODS}

Source of PHHs. PHHs were isolated from surgical liver resections as previously described (18). PHHs were obtained with informed consent approved by the ethics commission of Hannover Medical School (Ethik-Kommission der MHH, number 252-2008). Additionally, PHHs were commercially obtained (Lonza, Basel, Switzerland). Cryopreserved PHHs were thawed as recommended by the manufacturer.

Virus production and UV inactivation. Huh-7.5 cells were electroporated with HCV genomic RNA transcripts (Jc1 strain) $(40,41)$, and supernatants were harvested at 48,72 , and $96 \mathrm{~h}$ postelectroporation, filtered $\left(0.45-\mu \mathrm{m}\right.$ pores), pooled, aliquoted, and frozen at $-80^{\circ} \mathrm{C}$. A portion of this virus stock was UV inactivated in a 6-well dish with $1 \mathrm{ml}$ per well at $5 \mathrm{~J} / \mathrm{cm}^{2}$.

Generation of Huh-7.5 IRF1 cells. The IRF1 coding sequence (gBlock; IDT) was cloned into lentiviral vector pWPI-bla (Addgene) and confirmed by Sanger sequencing (GATC). Cotransfection of plasmids encoding vesicular stomatitis virus G protein (VSV-G) and HIV-1 Gag/Pol and plasmid pWPI-bla-IRF1 into HEK293T cells was performed using Lipofectamine 2000 (Invitrogen). Supernatants containing lentiviral pseudoparticles for transgene delivery were harvested at $24 \mathrm{~h}$ and $48 \mathrm{~h}$, pooled, filtered ( $0.45-\mu \mathrm{m}$ pores), and used to transduce $2 \times 10^{5}$ Huh-7.5 cells. At $72 \mathrm{~h}$ posttransduction, blasticidin $(10 \mu \mathrm{g} / \mathrm{ml})$ was added to media. For control purposes, Huh-7.5 cells were also transduced with pseudoparticles containing an empty pWPI-bla vector (no transgene expressed). After blasticidin addition, surviving cells were expanded via passaging for 14 days prior to freezing at $-150^{\circ} \mathrm{C}$ with $10 \%$ DMSO.

HCV infection and RNA isolation. PHH infections were performed at a multiplicity of infection (MOI) of 1, calculated using Huh-7.5 cells, and the same volume was used for treatment with UV-inactivated virus. At $4 \mathrm{~h}$ postinoculation, cells were washed with phosphate-buffered saline (PBS), and $1 \mathrm{ml}$ of fresh hepatocyte culture medium (HCM) was added per well (Lonza). At 6 or 72 hpi, supernatants were collected, and cells were lysed in $1 \mathrm{ml}$ TRIzol reagent (Invitrogen) or RA1 buffer supplemented with $\beta$-mercaptoethanol (Macherey-Nagel). All samples were stored at $-80^{\circ} \mathrm{C}$ until processing. Total RNA was extracted according to the manufacturer's instructions. For infection of Huh-7.5 empty vector-transduced cells, identical conditions were used except that Dulbecco's modified Eagle's medium (DMEM) was used instead of HCM and RNA extractions were performed using only the NucleoSpin RNA kit (Macherey-Nagel). Where ruxolitinib treatment is indicated, PHHs were pretreated with $10 \mu \mathrm{M}$ ruxolitinib on the day before HCV infection. For the rescue of viral particle production (Fig. $2 \mathrm{C}$ ), $10 \mu \mathrm{M}$ ruxolitinib was also added to HCM directly after infections.

Subgenomic replicon assays. Huh-7.5 cells expressing IRF1 or transduced with an empty lentivirus were electroporated with equal amounts $(2.5 \mu \mathrm{g})$ of a firefly luciferase (F-luc)-expressing subgenomic replicon RNA (NS3-NS5B, strain JFH-1) and seeded onto 12-well dishes. Additionally, empty control cells were treated with $1 \mu \mathrm{M} 2^{\prime} \mathrm{CMA}$ or DMSO. Cells were lysed at 4, 24, 48, 72, and $96 \mathrm{~h}$ postelectroporation in $350 \mu \mathrm{l}$ passive lysis buffer (Promega) per well and frozen at $-20^{\circ} \mathrm{C}$ until measurement of F-luc expression with a tube luminometer.

TCID $_{\mathbf{5 0}}$ and RT-qPCR. Viral titers in cellular supernatants and viral stocks used for infection experiments were quantified on Huh-7.5 cells using a limiting dilution assay as previously described (42). The limit of quantification (LOQ) was determined by the lowest, still evaluable result in the assay setup. Onestep RT-qPCR was performed to determine intracellular HCV RNA copy numbers using the LightCycler 480 RNA master hydrolysis probes kit (Roche) and an HCV-specific primer-probe set targeting the $5^{\prime}$ UTR. RT-qPCR was performed using a LightCycler 480 instrument (Roche). Ten RNA copies was the lowest number used to calculate the standard curve.

To determine the relative gene expression of selected cellular genes, $250 \mathrm{ng}$ of total cellular RNA was reverse transcribed using the TaKaRa reverse transcription (RT) kit. TaKaRa's SYBR Premix ExTaq II was used according to the manufacturer's instructions, with gene-specific primers. RT-qPCR was 
performed using a LightCycler 480 instrument (Roche). Changes in relative gene expression were calculated according to the $2^{-\Delta \Delta C T}$ method (43).

RNA-seq. Cellular RNAs were used to generate sequencing libraries using a ScriptSeqv2 kit (Illumina) and run on the Illumina HiSeq 2500 platform, and subsequent data analyses were performed using CLC Genomics Workbench (Qiagen, Aarhus, Denmark) (44). Mapping against the human reference genome (hg38) was performed for individual samples, and relative transcript expression was calculated from raw count data via normalization to gene length (RPKM) (45). Identification of differentially expressed genes (DEGs) was conducted by comparing raw count data, with calculation of the false discovery rate (FDR) $P$ values for multiple comparisons. Significant DEGs with low expression levels (FDR $P$ values of $<0.05$ with a final RPKM value of $<1$ ) were omitted from subsequent $G O, T F$, and pathway analyses.

GO enrichment analyses. GO analyses were performed using the GO Resource (http://geneontology .org/). ENSEMBL identifiers for DEGs were used as the input, and identification of significantly enriched GO categories was performed using the Panther classification system (http://pantherdb.org/). $P$ values for specific GO categories were generated after Bonferroni correction for multiple testing. Redundant GO terms were removed with REVIGO (46) (http://revigo.irb.hr), with the following settings: allowed similarity "tiny," GO term sizes database "Homo sapiens," and "SimRel" semantic similarity measure. The remaining terms were visualized in semantic similarity-based scatterplots using GraphPad Prism v9.0.

Canonical pathway analysis and transcriptional regulators. Pathway analyses were performed using ingenuity pathway analysis (Qiagen, Aarhus, Denmark) (47). Input data included ENSEMBL identifiers, FDR $P$ values, and fold change values for DEGs determined using CLC Genomics Workbench. Default settings were used for all categories except for species, which was set to human. Z-scores were calculated based on the expression fold change. IPA compares input data sets with the ingenuity knowledgebase, which represents a collection of published interactions of molecules. Upstream regulators are determined bioinformatically (http://pages.ingenuity.com/rs/ingenuity/images/0812\%20upstream_regulator_analysis_whitepaper.pdf). Analysis outputs were exported as Excel sheets or PDF files, and data were replotted with GraphPad and Adobe Illustrator. Disease-, cancer-, and non-hepatocyte-associated pathways were omitted from the presented diagrams.

Data availability. RNA-seq data generated in this study and subsequent downstream analyses, including the identification of DEGs, enriched GO categories, upstream TFs, and targeted pathways, were submitted to the NCBI GEO database under accession number GSE166428.

\section{ACKNOWLEDGMENTS}

We thank Charles Rice (Rockefeller University) for Huh-7.5 cells and anti-NS5A antibody 9E10. We also thank Robert Geffers, Michael Jarek, Maren Scharfe, and Sabin Bhuju (HZI Braunschweig, GMAK facility) for RNA-seq library preparation and generation of HiSeq 2500 raw data.

R.J.P.B. was supported by BMG grant 1-2516-FSB-416. T.P. was supported by a grant from the European Research Council, ERC-2011-StG_281473-(Virafront). T.P. was also funded by the Deutsche Forschungsgemeinschaft (DFG) (German Research Foundation) under Germany's Excellence Strategy-EXC 2155 Resist, project identifier 39087428.

TWINCORE is a joint venture between the Medical School Hannover (MHH) and the Helmholtz Centre for Infection Research (HZI), Hannover, Germany.

\section{REFERENCES}

1. Pietschmann T, Brown RJP. 2019. Hepatitis C virus. Trends Microbiol 27:379-380. https://doi.org/10.1016/j.tim.2019.01.001.

2. Vieyres G, Pietschmann T. 2020. Chapter 66: Tracking hepatitis C virus interactions with hepatic lipid metabolism: a hitchhiker's guide to solve remaining translational research challenges in hepatitis C. In Arias IM, Alter HJ, Boyer JL, Cohen DE, Shafritz DA, Thorgeirsson SS, Wolkoff AW (ed), The liver: biology and pathobiology, 6th ed. Wiley-Blackwell, Hoboken, NJ.

3. Vasallo C, Gastaminza P. 2015. Cellular stress responses in hepatitis $C$ virus infection: mastering a two-edged sword. Virus Res 209:100-117. https:// doi.org/10.1016/j.virusres.2015.03.013.

4. Gluchowski NL, Becuwe M, Walther TC, Farese RV, Jr. 2017. Lipid droplets and liver disease: from basic biology to clinical implications. Nat Rev Gastroenterol Hepatol 14:343-355. https://doi.org/10.1038/nrgastro.2017.32.

5. Horner SM, Gale M, Jr. 2013. Regulation of hepatic innate immunity by hepatitis C virus. Nat Med 19:879-888. https://doi.org/10.1038/nm.3253.

6. Bowie AG, Unterholzner L. 2008. Viral evasion and subversion of patternrecognition receptor signalling. Nat Rev Immunol 8:911-922. https://doi .org/10.1038/nri2436.

7. Schoggins JW, Wilson SJ, Panis M, Murphy MY, Jones CT, Bieniasz P, Rice CM. 2011. A diverse range of gene products are effectors of the type I interferon antiviral response. Nature 472:481-485. https://doi.org/10 .1038 /nature09907.
8. Woodhouse SD, Narayan R, Latham S, Lee S, Antrobus R, Gangadharan B, Luo S, Schroth GP, Klenerman P, Zitzmann N. 2010. Transcriptome sequencing, microarray, and proteomic analyses reveal cellular and metabolic impact of hepatitis $C$ virus infection in vitro. Hepatology 52:443-453. https://doi.org/ 10.1002/hep.23733.

9. Papic N, Maxwell Cl, Delker DA, Liu S, Heale BSE, Hagedorn CH. 2012. RNA-sequencing analysis of 5' capped RNAs identifies many new differentially expressed genes in acute hepatitis $C$ virus infection. Viruses 4:581-612. https://doi.org/10.3390/v4040581.

10. Blackham S, Baillie A, Al-Hababi F, Remlinger K, You S, Hamatake R, McGarvey MJ. 2010. Gene expression profiling indicates the roles of host oxidative stress, apoptosis, lipid metabolism, and intracellular transport genes in the replication of hepatitis C virus. J Virol 84:5404-5414. https:// doi.org/10.1128/JVI.02529-09.

11. Boldanova T, Suslov A, Heim MH, Necsulea A. 2017. Transcriptional response to hepatitis $C$ virus infection and interferon-alpha treatment in the human liver. EMBO Mol Med 9:816-834. https://doi.org/10.15252/ emmm.201607006.

12. Thomas E, Gonzalez VD, Li Q, Modi AA, Chen W, Noureddin M, Rotman Y, Liang TJ. 2012. HCV infection induces a unique hepatic innate immune response associated with robust production of type III interferons. Gastroenterology 142:978-988. https://doi.org/10.1053/j.gastro.2011.12.055. 
13. Park H, Serti E, Eke O, Muchmore B, Prokunina-Olsson L, Capone S, Folgori A, Rehermann B. 2012. IL-29 is the dominant type III interferon produced by hepatocytes during acute hepatitis $C$ virus infection. Hepatology 56:2060-2070. https://doi.org/10.1002/hep.25897.

14. Metz P, Dazert E, Ruggieri A, Mazur J, Kaderali L, Kaul A, Zeuge U, Windisch MP, Trippler M, Lohmann V, Binder M, Frese M, Bartenschlager R. 2012. Identification of type I and type II interferon-induced effectors controlling hepatitis C virus replication. Hepatology 56:2082-2093. https://doi.org/10.1002/hep.25908.

15. Sheahan T, Imanaka N, Marukian S, Dorner M, Liu P, Ploss A, Rice CM. 2014. Interferon lambda alleles predict innate antiviral immune responses and hepatitis C virus permissiveness. Cell Host Microbe 15:190-202. https://doi.org/10.1016/j.chom.2014.01.007.

16. Andrus L, Marukian S, Jones CT, Catanese MT, Sheahan TP, Schoggins JW, Barry WT, Dustin LB, Trehan K, Ploss A, Bhatia SN, Rice CM. 2011. Expression of paramyxovirus $V$ proteins promotes replication and spread of hepatitis $C$ virus in cultures of primary human fetal liver cells. Hepatology 54:1901-1912. https://doi.org/10.1002/hep.24557.

17. Wang Z, Gerstein M, Snyder M. 2009. RNA-Seq: a revolutionary tool for transcriptomics. Nat Rev Genet 10:57-63. https://doi.org/10.1038/nrg2484.

18. Kleine M, Riemer M, Krech T, DeTemple D, Jager MD, Lehner F, Manns MP, Klempnauer J, Borlak J, Bektas H, Vondran FW. 2014. Explanted diseased livers - a possible source of metabolic competent primary human hepatocytes. PLoS One 9:e101386. https://doi.org/10.1371/journal.pone.0101386.

19. Shulla A, Randall G. 2015. Spatiotemporal analysis of hepatitis C virus infection. PLoS Pathog 11:e1004758. https://doi.org/10.1371/journal.ppat .1004758.

20. Romero-Brey I, Merz A, Chiramel A, Lee JY, Chlanda P, Haselman U, Santarella-Mellwig R, Habermann A, Hoppe S, Kallis S, Walther P, Antony C, Krijnse-Locker J, Bartenschlager R. 2012. Three-dimensional architecture and biogenesis of membrane structures associated with hepatitis $C$ virus replication. PLoS Pathog 8:e1003056. https://doi.org/10.1371/ journal.ppat.1003056.

21. Blight KJ, McKeating JA, Rice CM. 2002. Highly permissive cell lines for subgenomic and genomic hepatitis C virus RNA replication. J Virol 76:13001-13014. https://doi.org/10.1128/jvi.76.24.13001-13014.2002.

22. Hubel P, Urban C, Bergant V, Schneider WM, Knauer B, Stukalov A, Scaturro P, Mann A, Brunotte L, Hoffmann HH, Schoggins JW, Schwemmle M, Mann M, Rice CM, Pichlmair A. 2019. A protein-interaction network of interferon-stimulated genes extends the innate immune system landscape. Nat Immunol 20:493-502. https://doi.org/10.1038/s41590-019-0323-3.

23. Panda D, Gjinaj E, Bachu M, Squire E, Novatt H, Ozato K, Rabin RL. 2019. IRF1 maintains optimal constitutive expression of antiviral genes and regulates the early antiviral response. Front Immunol 10:1019. https://doi .org/10.3389/fimmu.2019.01019.

24. Yamane D, Feng H, Rivera-Serrano EE, Selitsky SR, Hirai-Yuki A, Das A, McKnight KL, Misumi I, Hensley L, Lovell W, Gonzalez-Lopez O, Suzuki R, Matsuda M, Nakanishi H, Ohto-Nakanishi T, Hishiki T, Wauthier E, Oikawa T, Morita K, Reid LM, Sethupathy P, Kohara M, Whitmire JK, Lemon SM. 2019. Basal expression of interferon regulatory factor 1 drives intrinsic hepatocyte resistance to multiple RNA viruses. Nat Microbiol 4:1096-1104. https://doi.org/10.1038/s41564-019-0425-6.

25. Vassilaki N, Kalliampakou KI, Kotta-Loizou I, Befani C, Liakos P, Simos G, Mentis AF, Kalliaropoulos A, Doumba PP, Smirlis D, Foka P, Bauhofer O, Poenisch M, Windisch MP, Lee ME, Koskinas J, Bartenschlager R, Mavromara P. 2013. Low oxygen tension enhances hepatitis $C$ virus replication. J Virol 87:2935-2948. https://doi.org/10.1128/JVI.02534-12.

26. Wang GL, Jiang BH, Rue EA, Semenza GL. 1995. Hypoxia-inducible factor 1 is a basic-helix-loop-helix-PAS heterodimer regulated by cellular $\mathrm{O} 2$ tension. Proc Natl Acad Sci U S A 92:5510-5514. https://doi.org/10.1073/ pnas.92.12.5510.

27. Aizarani N, Saviano A, Sagar, Mailly L, Durand S, Herman JS, Pessaux P, Baumert TF, Grün D. 2019. A human liver cell atlas reveals heterogeneity and epithelial progenitors. Nature 572:199-204. https://doi.org/10.1038/ s41586-019-1373-2.

28. Meylan E, Curran J, Hofmann K, Moradpour D, Binder M, Bartenschlager R, Tschopp J. 2005. Cardif is an adaptor protein in the RIG-I antiviral pathway and is targeted by hepatitis C virus. Nature 437:1167-1172. https://doi .org/10.1038/nature04193.

29. Lauber C, Vieyres G, Terczynska-Dyla E, Anggakusuma, Dijkman R, Gad HH, Akhtar H, Geffers R, Vondran FWR, Thiel V, Kaderali L, Pietschmann T,
Hartmann R. 2015. Transcriptome analysis reveals a classical interferon signature induced by IFNlambda4 in human primary cells. Genes Immun 16:414-421. https://doi.org/10.1038/gene.2015.23.

30. Garcia-Sastre A. 2017. Ten strategies of interferon evasion by viruses. Cell Host Microbe 22:176-184. https://doi.org/10.1016/j.chom.2017.07.012.

31. Garaigorta U, Chisari FV. 2009. Hepatitis C virus blocks interferon effector function by inducing protein kinase R phosphorylation. Cell Host Microbe 6:513-522. https://doi.org/10.1016/j.chom.2009.11.004.

32. González-Almela E, Williams H, Sanz MA, Carrasco L. 2018. The initiation factors elF2, elF2A, elF2D, elF4A, and elF4G are not involved in translation driven by hepatitis C virus IRES in human cells. Front Microbiol 9:207. https://doi.org/10.3389/fmicb.2018.00207.

33. van Riggelen J, Yetil A, Felsher DW. 2010. MYC as a regulator of ribosome biogenesis and protein synthesis. Nat Rev Cancer 10:301-309. https://doi .org/10.1038/nrc2819.

34. Kim TW, Hong S, Lin Y, Murat E, Joo H, Kim T, Pascual V, Liu Y-J. 2016. Transcriptional repression of IFN regulatory factor 7 by MYC is critical for type I IFN production in human plasmacytoid dendritic cells. J Immunol 197:3348-3359. https://doi.org/10.4049/jimmunol.1502385.

35. Stöhr S, Costa R, Sandmann L, Westhaus S, Pfaender S, Anggakusuma, Dazert E, Meuleman P, Vondran FWR, Manns MP, Steinmann E, von Hahn T, Ciesek S. 2016. Host cell mTORC1 is required for HCV RNA replication. Gut 65:2017-2028. https://doi.org/10.1136/gutjnl-2014-308971.

36. Johri MK, Lashkari HV, Gupta D, Vedagiri D, Harshan KH. 2020. mTORC1 restricts hepatitis $C$ virus RNA replication through ULK1-mediated suppression of miR-122 and facilitates post-replication events. J Gen Virol 101:86-95. https://doi.org/10.1099/jgv.0.001356.

37. Levy G, Habib N, Guzzardi MA, Kitsberg D, Bomze D, Ezra E, Uygun BE, Uygun K, Trippler M, Schlaak JF, Shibolet O, Sklan EH, Cohen M, Timm J, Friedman N, Nahmias Y. 2016. Nuclear receptors control pro-viral and antiviral metabolic responses to hepatitis $C$ virus infection. Nat Chem Biol 12:1037-1045. https://doi.org/10.1038/nchembio.2193.

38. Brown RJP, Tegtmeyer B, Sheldon J, Khera T, Anggakusuma, Todt D, Vieyres G, Weller R, Joecks S, Zhang Y, Sake S, Bankwitz D, Welsch K, Ginkel C, Engelmann M, Gerold G, Steinmann E, Yuan Q, Ott M, Vondran FWR, Krey T, Ströh LJ, Miskey C, Ivics Z, Herder V, Baumgärtner W, Lauber C, Seifert M, Tarr AW, McClure CP, Randall G, Baktash Y, Ploss A, Thi VLD, Michailidis E, Saeed M, Verhoye L, Meuleman P, Goedecke N, Wirth D, Rice CM, Pietschmann T. 2020. Liver-expressed Cd302 and Cr1l limit hepatitis C virus cross-species transmission to mice. Sci Adv 6:eabd3233. https:// doi.org/10.1126/sciadv.abd3233.

39. Pfaender S, Brown RJ, Pietschmann T, Steinmann E. 2014. Natural reservoirs for homologs of hepatitis $C$ virus. Emerg Microbes Infect 3:e21. https://doi.org/10.1038/emi.2014.19.

40. Pietschmann T, Kaul A, Koutsoudakis G, Shavinskaya A, Kallis S, Steinmann E, Abid K, Negro F, Dreux M, Cosset FL, Bartenschlager R. 2006. Construction and characterization of infectious intragenotypic and intergenotypic hepatitis C virus chimeras. Proc Natl Acad Sci U S A 103:7408-7413. https://doi.org/10.1073/pnas.0504877103.

41. Haid S, Windisch MP, Bartenschlager R, Pietschmann T. 2010. Mouse-specific residues of claudin- 1 limit hepatitis $C$ virus genotype 2 a infection in a human hepatocyte cell line. J Virol 84:964-975. https://doi.org/10.1128/ JVI.01504-09.

42. Vieyres G, Pietschmann T. 2013. Entry and replication of recombinant hepatitis $C$ viruses in cell culture. Methods 59:233-248. https://doi.org/10 .1016/j.ymeth.2012.09.005.

43. Livak KJ, Schmittgen TD. 2001. Analysis of relative gene expression data using real-time quantitative PCR and the 2(-Delta Delta $C(T))$ method. Methods 25:402-408. https://doi.org/10.1006/meth.2001.1262.

44. Baruzzo G, Hayer KE, Kim EJ, Di Camillo B, FitzGerald GA, Grant GR. 2017. Simulation-based comprehensive benchmarking of RNA-seq aligners. Nat Methods 14:135-139. https://doi.org/10.1038/nmeth.4106.

45. Mortazavi A, Williams BA, McCue K, Schaeffer L, Wold B. 2008. Mapping and quantifying mammalian transcriptomes by RNA-Seq. Nat Methods 5:621-628. https://doi.org/10.1038/nmeth.1226.

46. Supek F, Bošnjak M, Škunca N, Šmuc T. 2011. REVIGO summarizes and visualizes long lists of gene ontology terms. PLoS One 6:e21800. https://doi .org/10.1371/journal.pone.0021800.

47. Kramer A, Green J, Pollard J, Jr, Tugendreich S. 2014. Causal analysis approaches in ingenuity pathway analysis. Bioinformatics 30:523-530. https://doi.org/10.1093/bioinformatics/btt703. 